\title{
Using input minimization to train a cerebellar model to simulate regulation of smooth pursuit
}

\author{
Fredrick H. Rothganger • Thomas J. Anastasio
}

Received: 18 August 2008 / Accepted: 2 October 2009 / Published online: 24 November 2009

(c) The Author(s) 2009. This article is published with open access at Springerlink.com

\begin{abstract}
Cerebellar learning appears to be driven by motor error, but whether or not error signals are provided by climbing fibers $(\mathrm{CFs})$ remains a matter of controversy. Here we show that a model of the cerebellum can be trained to simulate the regulation of smooth pursuit eye movements by minimizing its inputs from parallel fibers (PFs), which carry various signals including error and efference copy. The CF spikes act as "learn now" signals. The model can be trained to simulate the regulation of smooth pursuit of visual objects following circular or complex trajectories and provides insight into how Purkinje cells might encode pursuit parameters. In minimizing both error and efference copy, the model demonstrates how cerebellar learning through PF input minimization (InMin) can make movements more accurate and more efficient. An experimental test is derived that would distinguish InMin from other models of cerebellar learning which assume that CFs carry error signals.
\end{abstract}

Keywords Cerebellum - Smooth pursuit . Computational model $\cdot$ Learning

F. H. Rothganger

Sandia National Laboratories, Albuquerque, NM 87123, USA

e-mail: frothga@ sandia.gov

URL: http://www.sandia.gov

T. J. Anastasio

Department of Molecular and Integrative Physiology, University

of Illinois at Urbana-Champaign, Urbana, IL 61822, USA

T. J. Anastasio ( $\square)$

Beckman Institute for Advanced Science and Technology, University of Illinois at Urbana-Champaign, Urbana, IL 61822, USA

e-mail: tja@illinois.edu

URL: http://csn.beckman.uiuc.edu/

\section{List of abbreviations}

$A \quad$ Amplitude of input sine waves

$B_{\mathrm{c}} \quad$ Base firing rate of climbing fibers ( $\mathrm{CFs}$ )

$b \quad$ Bin number for $\mathrm{CF}$ spike histogram

c CF signal

$\dot{\mathbf{C}}$ Total velocity command from frontal eye fields (FEF)

C Integrated velocity (position) command from FEF

$d \quad$ Delay due to visual system processing

$\mathbf{e}, \dot{\mathbf{e}} \quad$ Eye position and velocity

$f \quad$ Base frequency of input sine waves

$g_{\mathrm{r}} \quad$ Gain for smooth eye movement (r)

$g_{\mathrm{s}} \quad$ Gain for saccades (s)

g Purkinje cell (PC) gains specific to a microzone (MZ)

$g_{i} \quad$ Gain of individual PC

$\Delta \mathbf{g} \quad$ Perturbation specific to an MZ

$\|\Delta \mathbf{g}\| \quad$ Scale of perturbations

$\gamma_{f} \quad$ Decay rate for brainstem integrator

$\gamma_{1} \quad$ Decay rate for long parallel fiber (PF) activity integrator

$\gamma_{\mathrm{s}} \quad$ Decay rate for short PF activity integrator

h Activity vector of all PFs assigned to an MZ

$\bar{h}_{1} \quad$ Smoothed PF activity from the long integrator

$\bar{h}_{\mathrm{s}} \quad$ Smoothed PF activity from the short integrator

$\Delta \bar{h} \quad$ Change in PF activity

$i \quad$ Generic index

$j \quad$ Index of winning PC in self-organizing map (SOM) learning

$L_{\mathrm{c}} \quad$ Competitive (SOM) learning rate

$L_{\mathrm{p}} \quad$ Perturbative learning rate

$L_{\mathrm{d}} \quad$ Delta rule learning rate

M Masking matrix for delta-rule learning

m Motor command fed into eye plant

$m \quad$ Combined output of a single cerebellar MZ 
$n \quad$ Quantities of various things (PCs, PFs, etc)

$\mathbf{o}, \dot{\mathbf{o}}$ Object position and velocity

$\phi \quad$ Phase lead of input sine waves

p Output of the PCs in a specific MZ (before scaling by $\mathbf{g})$

P Combined output of cerebellum following push-pull processing

$\mathbf{r}, \dot{\mathbf{r}}$ Retinal position (error), retinal velocity (slip error)

$\dot{\mathbf{s}} \quad$ Saccade velocity

$\Delta t \quad$ Duration of one discrete time-step in simulation

$T_{\Delta} \quad$ PF activity change threshold

$T_{\mathrm{s}} \quad$ Saccade threshold

$\tau_{\mathrm{e}} \quad$ Eye plant time constant; also used to scale velocity command $\dot{\mathbf{C}}$

$\mathrm{u} \quad$ Random number in $[0,1]$ drawn from uniform distribution

W PF to PC weight matrix

$\mathbf{x}, \dot{\mathbf{x}}$ Position and velocity sensitivity vectors

\section{Introduction}

The cerebellum is critical for the adaptive regulation of movement in vertebrate animals and has been studied extensively in the context of eye movement control. Purkinje cells (PCs), which are the principle and the efferent cells of the cerebellar cortex, receive excitatory input only from parallel fibers (PFs) and climbing fibers (CFs) (Eccles et al. 1967; Llinás and Walton 1990; Butler and Hodos 1996). Cerebellar learning is thought to involve adaptive plasticity of the synapses between PFs and PCs. Most models of cerebellar learning of eye movement control (e.g., Fujita 1982; Kettner et al. 1997; Ito 2000; Yamamoto et al. 2002) follow the Marr/Albus paradigm (Marr 1969; Albus 1971), in which CFs provide error signals that determine the values of adaptive changes made to parallel-Purkinje synapses. Whether or not CFs carry error signals of sufficient precision and temporal resolution to drive cerebellar learning is an unresolved issue. The purpose of this article is to explore an alternative to the Marr/Albus paradigm, in the context of a model of the smooth pursuit system, which produces cerebellar learning without CF error signals.

The role of the CF signal in cerebellar learning is enigmatic (Simpson et al. 1996; Gibson et al. 2004; Kitazawa and Wolpert 2005). Specifically, the relationship between CF spike discharge and adaptive modification of smooth pursuit is still unclear. If CFs do guide cerebellar learning in the context of smooth pursuit, then their discharge should be robustly modulated by the retinal slip error signal, which is the difference in velocity between the eye (fovea) and the visual object being pursued. CFs originate in the inferior olive, but their discharge can be observed indirectly from its effects on
PCs. Each CF spike causes the PCs that receive it to produce a prolonged, multi-peaked, complex spike. Complex spikes are easily distinguished from the conventional action potentials (simple spikes) caused by PF input (Eccles et al. 1967; Ito 1984). If the Marr/Albus paradigm is correct, then PC complex spike rates should be well correlated with retinal slip. Evidence for this is inconclusive.

Purkinje cell complex spikes occur at rates less than $1 \mathrm{~Hz}$, which have been termed "ultra-low" (Kuroda et al. 2001), and their firing pattern in awake, behaving monkeys is random (Keating and Thach 1995, 1997). Modulation of the low-frequency and random complex spikes is difficult to discern. Some studies in monkeys reported that the complex spikes of floccular PCs are modulated by retinal slip (Stone and Lisberger 1990b). Later work revealed that the complex spike modulation of these cells is weak ( $2 \%$ of simple spike modulation) and discernable only after considerable temporal averaging (Kobayashi et al. 1998). Studies of pursuit by monkeys of transient targets show that the complex spike discharge of floccular PCs, when averaged over many (50 to 200) trails, can show modulations due to the onset of image motion (Kahlon and Lisberger 2000). These modulations were considered to be retinal slip error signals, but they were not consistently related to learning-induced changes in pursuit or in PC simple spike discharge (ibid). Other studies have determined that $\mathrm{CF}$ and complex spike discharge is better correlated with eye velocity and other motor variables than with retinal slip error during oculomotor behaviors (Kobayashi et al. 1998; Simpson et al. 2002). Available data leave open the possibility that CFs do not carry error signals. Input minimization (InMin) offers an alternative view of cerebellar learning that is consistent with this possibility.

The flocculus and ventral paraflocculus (the floccular complex) of the cerebellum play a major role in smooth pursuit eye movement control (Zee et al. 1981; Rambold et al. 2002). Signals related to object and eye movement are transmitted to the cerebellum over mossy fibers, which contact granule cells (GCs), which in turn give rise to PFs. Neurons in the dorsolateral pontine nucleus (DLPN) project as mossy fibers to the floccular complex (Brodal 1979, 1982; Glickstein et al. 1994). DLPN neurons encode various quantities associated with eye and visual object motion including retinal slip (Mustari et al. 1988; Suzuki et al. 1990; Takemura et al. 2001). Thus, PFs carry retinal slip error signals, along with many other types of signals, and it is possible that the cerebellum could learn to reduce retinal slip error by adapting its regulation of ongoing pursuit behavior so as to minimize its overall input from PFs.

Parallel fibers also carry copies of motor commands (efference copy signals). Neurons in the nucleus reticularis tegmenti pontis (NRTP) encode saccade commands (Crandall and Keller 1985) and project as mossy fibers to various cerebellar regions including the floccular complex (Brodal 1979, 1982; 
Glickstein et al. 1994). Such signals could serve as efference copy of commands for the catch-up saccades that occur when pursuit falls short. The generation and execution of these commands require an extra effort that is not needed when pursuit is accurate. Thus, PFs carry signals such as object and eye movement signals, which generally cannot be minimized through improved performance, but also carry error and catch-up saccade efference copy signals that can be reduced through improved performance. Therefore, minimizing overall PF activity would simultaneously reduce retinal slip error and catch-up saccade effort, thereby improving both the accuracy and the efficiency of pursuit eye movements.

An algorithm based on InMin has been used to train a model cerebellum to reduce retinal slip error and simulate adaptation of the vestibulo-ocular reflex (Anastasio 2001a). The purpose of this study is to further explore InMin, in the context of a model of cerebellar regulation of smooth pursuit eye movements. Here, we improve the algorithm and show how InMin can be used to reduce both retinal slip error and catch-up saccade effort. The response properties of simulated PCs compare well with real data, and the model provides a new hypothesis concerning how PCs might encode pursuit variables. Finally, we derive an experimental test of PC responses that could be used to determine whether actual cerebellar adaptation is more consistent with InMin or with Marr/Albus.

\section{Methods}

The pursuit model is intended to be similar to current models except that the learning paradigm is InMin rather than Marr/Albus. Our general aim is to demonstrate the viability of InMin as a cerebellar learning algorithm. Our specific aim is to simulate the data collected by Kettner and colleagues on predictive smooth pursuit of objects moving along two-dimensional trajectories (Kettner et al. 1996; Leung and Kettner 1997) and on the responses of PCs in the floccular complex during such pursuit (Leung et al. 2000; Suh et al. 2000). Our model is similar to the pursuit model developed by Kettner et al. (1997) in that the cerebellar component must learn to overcome a delay in its visual input and must therefore predict the trajectory of the visual object in order to pursue it accurately. Our model will also generate catch-up saccades when pursuit falls short of the pursued object (ibid). Our model differs from that of Kettner et al. (1997) in that it also includes a direct pursuit pathway through the cortex, which bypasses the cerebellum (see Krauzlis 2004 for review). This pathway has been included in other recent models of the cerebellar regulation of smooth pursuit (Kuroda et al. 2001; Yamamoto et al. 2002). Again, the main difference between our model and other recent models of the cerebellar regulation of pursuit is that the learning paradigm is InMin rather than Marr/Albus.

\subsection{Model structure}

The overall structure of the model is diagrammed in Fig. 1 . Many of the variables in the model refer to angles in a threedimensional coordinate system attached to the optical center of the eye, with the $x$-axis pointing to the right, the $y$-axis pointing down, and the $z$-axis pointing forward. For simplicity, we treat the eye as rotating exactly around its optical center and treat the target object as a dot formed by a laser beam emanating from the origin and striking a spherical screen. The input to the model is the object direction vector $\mathbf{o}=\left[o_{\mathrm{h}}, o_{\mathrm{v}}\right]^{\mathrm{T}}$, where $o_{\mathrm{h}}$ and $o_{\mathrm{v}}$ are angles in the horizontal and vertical planes ( $\mathrm{T}$ is the transpose operator, and all vectors are columns by convention). Thus $o_{\mathrm{h}}$ is rotation around the $y$-axis made by the projection of $\mathbf{o}$ into the $x z$ plane. Similarly, $o_{\mathrm{v}}$ is rotation around the $x$-axis made by the projection of $\mathbf{o}$ into the $y z$ plane. These are essentially Euler angles, but the use of projection in their definition evades any

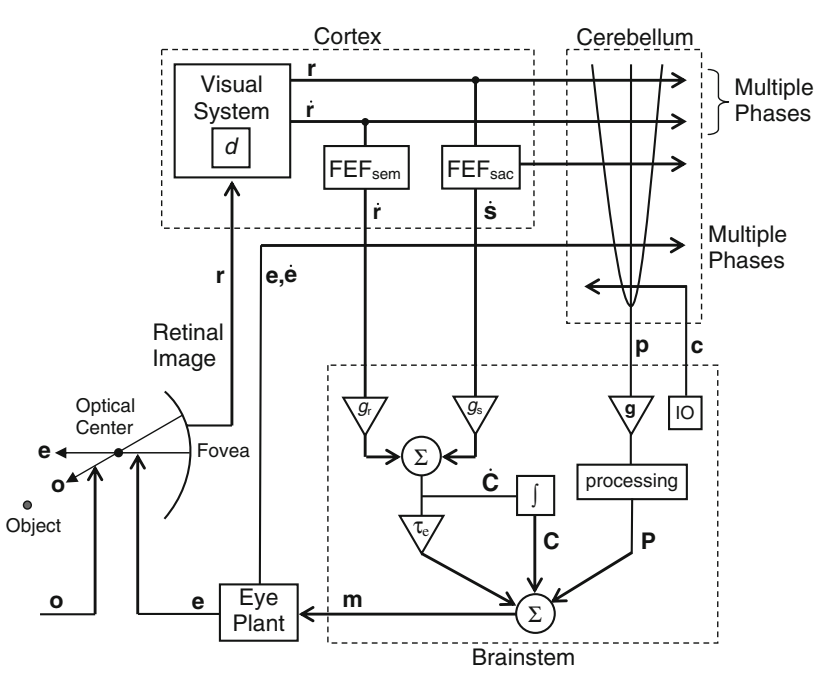

Fig. 1 Schematic of the pursuit model. The eye points in direction $\mathbf{e}$ while the object is located at position $\mathbf{0}$. The visual system receives the retinal (error) position $\mathbf{r}$ signal, processes it, incurring delay $d$ seconds, and then passes it on in the form of various phases of retinal position and slip velocity $(\dot{\mathbf{r}})$. The frontal eye fields (FEF) controlling smooth eye movement (FEFsem) and saccades (FEFsac), respectively, send retinal slip and saccade velocity $\dot{\mathbf{s}}$ commands to the brainstem. These signals sum via retinal slip $\left(g_{\mathrm{r}}\right)$ and saccade $\left(g_{\mathrm{s}}\right)$ gains into a velocity command $(\dot{\mathbf{C}})$. This command reaches the motoneurons both as a scaled velocity with gain $\tau_{\mathrm{e}}$ and as an integrated position command $\mathbf{C}$. Finally, the motor command $(\mathbf{m})$ controls eye position. The cerebellum receives eye movement (e and $\dot{\mathbf{e}}$ ) signals, via proprioception and/or efference copy, and saccade-command efference copy. The visual system also sends its outputs to the cerebellum. The responses $\mathbf{p}$ of Purkinje cells are weighted by gains $\mathbf{g}$ and processed (see text and Figs. 2 and 3) to produce the cerebellar command $\mathbf{P}$. The inferior olive (IO) outputs climbing fiber spikes (c) to the cerebellum 
order dependency between them. Direction vectors for eye $\mathbf{e}$, retinal error $\mathbf{r}$, and saccade command $\mathbf{s}$ are defined similarly. The term "position" refers to a direction vector, while "velocity" refers to the rate of change of a direction vector.

Following Kettner et al. (1997), the elements of o vary sinusoidally with time, at frequencies that are integer multiples of a base frequency. The period of one cycle of the resulting two-dimensional waveform is called an "epoch." All the results presented here have base frequency $f=1 / 3 \mathrm{~Hz}$, with the corresponding epoch lasting $3 \mathrm{~s}$. The name of an input pattern indicates the direction and frequency multiples of its component sine waves. We used two standard patterns. One is the H3V2 pattern, which produces a trajectory resembling a pretzel:

$\left[\begin{array}{c}o_{\mathrm{h}} \\ o_{\mathrm{v}}\end{array}\right](t)=\left[\begin{array}{l}A \sin (3 f t \cdot 2 \pi+\phi) \\ A \sin (2 f t \cdot 2 \pi+\phi)\end{array}\right]$,

where $t$ is time in seconds, $A=0.1$ radians $\approx 5^{\circ}$ is the amplitude, and $\phi$ is a phase lead (described later). The other input pattern is $\mathrm{H} 2 \mathrm{~V} 2$, with the vertical component phase lagging the horizontal by $\pi / 2$ to produce a counter-clockwise circular motion:

$\left[\begin{array}{l}o_{\mathrm{h}} \\ o_{\mathrm{v}}\end{array}\right](t)=\left[\begin{array}{l}A \sin (2 f t \cdot 2 \pi+\phi) \\ A \sin \left(2 f t \cdot 2 \pi+\phi-\frac{\pi}{2}\right)\end{array}\right]$.

Eye position $\mathbf{e}$ is the vector from the fovea to the optical center. Object position is subtracted from eye position to compute retinal position (error) $\mathbf{r}$. The retinal position error signal is sent to the visual system for processing which yields the retinal slip velocity error signal $\dot{\mathbf{r}}$. The model visual system is meant to represent the cortical areas that are involved in pursuit processing, such as the medial superior temporal (MST) region (Newsome et al. 1988; Kawano et al. 1994; Takemura et al. 2001). The delay $d$ that the pursuit system must overcome is associated with visual processing.

The visual system feeds three parallel pathways that each contribute to pursuit. One of these is the cerebellar pathway (described below). The two other pathways include the frontal eye fields (FEF). FEFsac is the well-known saccaderelated portion of the FEF (see Goldberg and Segraves 1989; Sparks and Barton 1993 for reviews). In the model, production of a saccade command depends both on retinal position error $\mathbf{r}$ and on time. The FEFsac module enters a state of preparation to issue a saccade command whenever $\|\mathbf{r}\|>T_{\mathrm{s}}$, where $T_{\mathrm{S}}$ is the saccade threshold and $\|\cdot\|$ is the vector 2norm. FEFsac issues the saccade command after a delay of at least $100 \mathrm{~ms}$, to reflect the visual processing delay, and at least $200 \mathrm{~ms}$ after the start of the previous saccade, to reflect the saccadic refractory period. A saccade command occurs over 4 time-steps and has the following three components: a large $\dot{\mathbf{s}}$ pulse in the desired direction for 1 time-step, a 0 command for 2 time-steps, and a large $\dot{\mathbf{s}}$ pulse in the opposite direction for 1 time-step ( $\dot{\mathbf{s}}$ is the saccade command velocity vector). The saccade command is weighted by saccade gain $g_{\mathrm{s}}$ and applied as a velocity command to the brainstem premotor pathway (to be described below). While the details of saccade generation are beyond the focus of this study, the use of both drive and brake pulses is meant to simulate the actual interplay of these two command components (see Takagi et al. 1998; Goffart et al. 2003 for reviews). The drive and brake pulses are sized so that, when integrated in the model brainstem, their difference equals $70 \%$ of the desired amount of eye position change. Saccadic undershooting in the model is meant to reflect that observed experimentally (Guitton et al. 2003).

FEFsem is the more recently described smooth eye movement portion of the FEF. Lesions of FEFsem impair smooth pursuit (Lynch 1987; Keating 1991, 1993; Shi et al. 1998), while electric stimulation of FEFsem produces smooth eye movements (Tain and Lynch 1996a; Tanaka and Lisberger 2001, 2002). FEFsem receives input from MST (Tain and Lynch 1996b), and the activities of neurons in FEFsem are modulated during pursuit by a combination of retinal slip and eye velocity (MacAvoy et al. 1991; Tanaka and Fukushima 1998; Tanaka and Lisberger 2002). This response characteristic is consistent with the hypothesis that FEFsem is the controller of a servomechanism that reduces retinal slip velocity by using it as a command to drive eye velocity. FEFsem acts as a pursuit servo in the model. It simply weights the retinal slip error signal $\dot{\mathbf{r}}$ by its forward loop gain $g_{\mathrm{r}}$ and applies this as an eye velocity command to the brainstem pre-motor pathway.

FEFsem and FEFsac work in complementary fashion in the model. FEFsac samples the retinal position error signal $\mathbf{r}$ from the visual system and generates a catch-up saccadic eye velocity command $\dot{\mathbf{s}}$ whenever $\mathbf{r}$ exceeds a threshold. FEFsem receives the retinal velocity error (retinal slip) signal $\dot{\mathbf{r}}$ and reduces this error by using it as a continuous eye velocity command. Thus, FEFsac and FEFsem act to keep the eye on the pursued object by reducing retinal position and velocity error, respectively. The eye velocity commands generated by FEFsem and FEFsac are weighted by gains $g_{\mathrm{r}}$ and $g_{\mathrm{s}}$, respectively, and are then sent over the direct and integrated pre-motor brainstem pathways to the eye plant (to be described below).

The third pursuit pathway represented in the model passes through the cerebellum. The flocculus and the ventral paraflocculus (the floccular complex) of the cerebellum are critical for the control of smooth eye movements including pursuit. Lesions of the floccular complex impair pursuit (Zee et al. 1981; Rambold et al. 2002), while electric stimulation in this region produces smooth eye movements (Ron and Robinson 1973; Miles and Fuller 1975). The floccular complex receives a major input from the DLPN (Brodal 1979, 1982; Glickstein et al. 1994). DLPN neurons vary greatly in their response properties. Some encode retinal 
slip error, while others encode quantities associated with eye and visual object motion (Mustari et al. 1988; Suzuki et al. 1990; Takemura et al. 2001). The model cerebellum receives retinal error $(\mathbf{r}, \dot{\mathbf{r}})$ and eye movement $(\mathbf{e}, \dot{\mathbf{e}})$ inputs, both with some additional phase shifts (see next section). In the model, the eye movement signals are actual eye position and velocity, but in the brain these signals could be derived from proprioseption, efference copy, or both. The model cerebellum also receives efference copy of the saccade velocity command $\dot{\mathbf{s}}$.

The discharge rate modulations of real PCs from the floccular complex are correlated mostly with eye position and velocity during smooth pursuit (Stone and Lisberger 1990a; Leung et al. 2000; Suh et al. 2000; Takemura et al. 2001; Kettner et al. 2002). The eye position signal is generated by the brainstem neural integrator in concert with the floccular complex (Zee et al. 1981; Chelazzi et al. 1990). The dynamics of this interaction are complex (e.g., Anastasio and Gad 2007; Barreiro et al. 2008) and beyond the focus of this study. Since PC commands already carry an eye position component, they are added to the FEFsem and FEFsac commands after the parallel direct and integrated pathways (described next).

In the model, the brainstem pre-motor controller is composed of parallel direct and integrated pathways to the eye plant (Robinson 1981). A perfect integrator in parallel with a direct pathway having gain $\tau_{\mathrm{e}}$ will compensate a first-order eye plant having time constant $\tau_{\mathrm{e}}$ (ibid). We use a first-order eye plant described by

$\mathbf{m}(t)=\tau_{\mathrm{e}} \dot{\mathbf{e}}(t)+\mathbf{e}(t)$,

where $\mathbf{e}$ is the eye position vector, $\mathbf{m}$ is the motoneuron command vector, $\tau_{\mathrm{e}}$ is the time constant of the eye plant, and $t$ is continuous time. We use the following simple, discrete approximation to compute updates to Eq. 3 :

$\mathbf{e} \leftarrow\left(1-\frac{\Delta t}{\tau_{\mathrm{e}}}\right) \mathbf{e}+\frac{\Delta t}{\tau_{\mathrm{e}}} \mathbf{m}$,

where $\Delta t=10 \mathrm{~ms}$ is the size of the discrete time-step. The combined FEFsem and FEFsac velocity commands $\dot{\mathbf{C}}$ is

$\dot{\mathbf{C}}=\left(g_{r} \dot{\mathbf{r}}+g_{s} \dot{\mathbf{s}}\right) \Delta t$,

where $\dot{\mathbf{r}}$ and $\dot{\mathbf{s}}$ are the retinal slip and saccade velocity vectors, and $g_{\mathrm{r}}$ and $g_{\mathrm{s}}$ are the gains of the pursuit-servo (forward-loop gain) and the saccade command, respectively (see above). In Eq. 5, $\Delta t$ converts units from radians/s to radians/time-step. The combined FEFsem and FEFsac position command is generated through discrete updates of:

$\mathbf{C} \leftarrow \gamma_{f} \mathbf{C}+\dot{\mathbf{C}}$

With $\gamma_{\rho}=0.9995$, Eq. 6 acts as a discrete version of a leaky (imperfect or lossy) integrator with a time constant of about $20 \mathrm{~s}$. It represents the oculomotor neural integrator, the time constant of which is about $20 \mathrm{~s}$ in humans and cats (Becker and Klein 1973; Robinson 1974). Since the PC responses already have both velocity and position components (see above), the model motoneuron command vector is computed as

$\mathbf{m}=\mathbf{C}+\tau_{\mathrm{e}} \dot{\mathbf{C}}+\mathbf{P}$,

where $\mathbf{P}=\left(P_{\mathrm{h}}, P_{\mathrm{v}}\right)$ is the command derived from the model $\mathrm{PC}$ responses (described below). Passing the combined FEFsem and FEFsac commands through the direct and integrated pathways ensures that they compensate plant dynamics. The cerebellar component learns to do so by differentially combining the eye movement inputs (of various phases) to individual PCs and by adjusting the PC output weights (see Sect. 2.3).

\subsection{Construction of the model cerebellum}

The cerebellar component of the model is shown in Fig. 2. It is organized according to microzones (MZs). Each MZ consists of the set of 12 PCs innervated by a single CF (Andersson and Oscarsson 1978; Oscarsson 1979; Voogd and Bigaré 1980). The cerebellar component comprises four MZs, arranged as opposed pairs controlling the two principal dimensions (horizontal and vertical) and operating in push-pull. For simplicity, the non-cerebellar components of the model are not arranged in push-pull, so the exclusively inhibitory output of real PCs is represented in the model as excitation or inhibition by the PCs in opposing MZs.

All the MZs receive the horizontal and vertical components of $\mathbf{e}$, which are presented at 12 different phase leads relative to eye position: $0, \pi / 12,2 \pi / 12, \ldots, 11 \pi / 12$. For

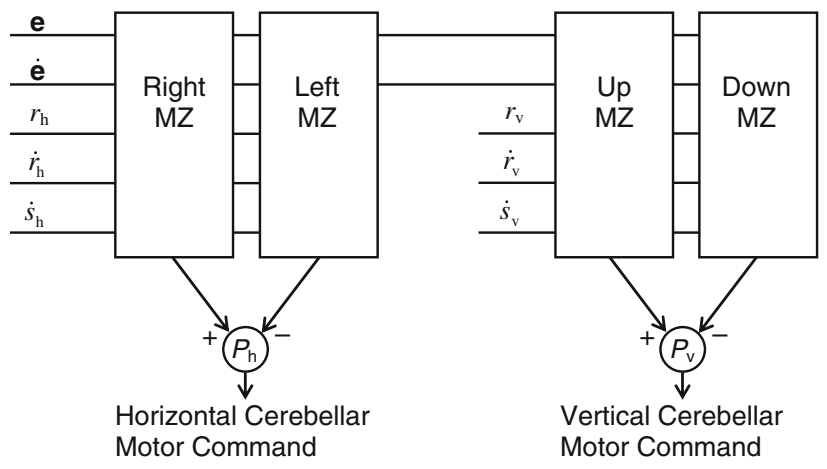

Fig. 2 Schematic of the model cerebellum, which consists of four microzones (MZs). In each dimension (horizontal or vertical), a pair of MZs operates in push-pull to provide the positive and negative components of the command. The eye position $(\mathbf{e})$ and velocity $(\dot{\mathbf{e}})$ signals, along with signals at other phases, are sent as inputs to all MZs (right/left and up/down). The retinal position error (r), retinal velocity (slip) error $(\dot{\mathbf{r}})$, and saccade velocity $(\dot{\mathbf{s}})$ signals are split up into horizontal and vertical components and are sent as inputs to dimension-specific MZs (right/left or up/down). The responses of individual PCs in each MZ are weighted by the elements of $\mathbf{g}$ (see Fig. 3) and combined in push-pull to produce cerebellar commands $P_{\mathrm{h}}$ and $P_{\mathrm{v}}$. The weights in $\mathbf{g}$ are adapted through perturbative reinforcement learning 
simplicity, the phase leads are emulated using a range of delays on two basic signals: $\mathbf{e}$, and $\dot{\mathbf{e}}$ computed from $\mathbf{e}$ by central differences. Given a perfectly sinusoidal signal, inverting and delaying by an angle $\alpha$ is equivalent to a phase lead of $\pi-\alpha$. Thus, all MZs in the model receive a signal that is in phase with eye position e, five signals spaced evenly in phase between eye position and eye velocity, a signal that is in phase with eye velocity $\dot{\mathbf{e}}$, and five signals spaced evenly in phase between eye velocity and eye acceleration (but not including eye acceleration). An analogous set of 12 signals, spaced evenly in phase from 0 to $11 \pi / 12$ with respect to position, is also available for retinal error (note that this set includes $\mathbf{r}$ and $\dot{\mathbf{r}}$ ). This spread in phase represents the phase variability present in the pursuit-related inputs to the cerebellum (Mustari et al. 1988; Suzuki et al. 1990; Takemura et al. 2001). Phase spreading does not apply to the transient saccadic efference copy signal $\dot{\mathbf{s}}$. Note that all MZs receive the eye-related signals for both dimensions, horizontal and vertical. This mixing of dimension has interesting consequences for model performance and enables the model to simulate the responses of real PCs (see Sect. 3).

Unlike the eye-related signals, the retinal error and saccadic efference copy signals are segregated by dimension. An MZ learns most efficiently when the dimension of the retinal error signals it receives is the same as the dimension of eye rotation it controls. The model also learns, albeit more slowly and with less accurate results, when its retinal error inputs and command outputs are connected in more complicated ways. In particular, it can learn even when its MZs receive retinal error signals of mixed dimension, or when all MZs control both dimensions of eye rotation. For simplicity, in the simulations the MZs will receive retinal error and saccadic efference copy signals and will send eye movement commands that are segregated by dimension.

Figure 3 shows the construction of an individual MZ. The inputs arrive on model mossy fibers. As in models that are consistent with the Marr/Albus paradigm, our model implements an expansive re-coding of the mossy fiber signals by the GCs. Each dimension (horizontal and vertical) of each signal has one mossy fiber to indicate its activity in the positive range and another one to indicate its activity in the negative range. There are 25 input signals ( $\dot{\mathbf{s}}$, plus six phases each of $\mathbf{e}, \dot{\mathbf{e}}, \mathbf{r}$, and $\dot{\mathbf{r}}$ ), times two dimensions (horizontal and vertical), times two signs ( + and - ), for a total of 100 mossy fibers. In order to implement the expansive re-coding, a single mossy fiber contacts several GCs, and every GC receives exactly one mossy fiber. The GCs are binary threshold elements. Each GC outputs a 1 at any time-step in which its mossy fiber input exceeds its threshold and outputs a 0 otherwise. Each GC in the group that receives a given mossy fiber signal has a different threshold, and the thresholds are distributed evenly across the range of expected values.

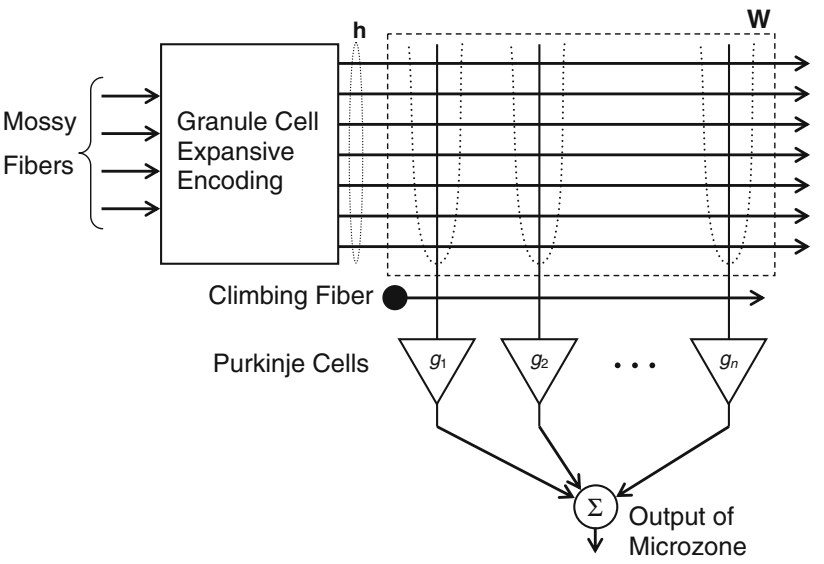

Fig. 3 Structure of one microzone. Signals arrive on mossy fibers. The granule cells recode the signals into a sparse form and deliver them on parallel fibers (h) to the Purkinje cells (p). The Purkinje cells respond to these signals according to their synaptic weights $(\mathbf{W}): \mathbf{p}=\mathbf{W h}$. The individual PC responses are weighted by gains $g_{i}$ (in $\mathbf{g}$ ) and combined to form the output of the microzone $(m): m=\mathbf{g}^{\mathrm{T}} \mathbf{p}$

Each GC gives rise to a PF, and the GC and PF activities are the same. The vector of the activities of the PFs that impinge on a given $\mathrm{MZ}$ is designated as $\mathbf{h}$. Considering the GC thresholds, the PF activities associated with a given input component $x$ are:

$h_{x i}^{+}=x>\frac{T_{x}(i-1)}{\left(n_{x}-1\right)}$ and $h_{x i}^{-}=x<\frac{-T_{x}(i-1)}{\left(n_{x}-1\right)}$,

$$
i=1, \ldots, n_{x}
$$

where $h_{x i}^{+}$and $h_{x i}^{-}$are the elements $i$ of $\mathbf{h}$ associated with mossy fiber $x$ representing its positive and negative ranges, respectively, $T_{x}$ is the maximum expected absolute value for the given input component, and $n_{x}$ is the number of GCs devoted to encoding the positive or negative values of mossy fiber $x$.

The thresholds used to determine the GC (and so the PF) activities depend on the specific input component. Table 1 shows the major signals along with their associated parameters. The thresholds for eye (e) related signals and retinal slip (r) related signals are based on the amplitude of the object motion $\mathbf{o}$. The threshold for the saccade signal $\dot{\mathbf{s}}$ is based on the maximum saccade velocity observed in monkeys: between 800 and 1000 degrees/second (Fuchs 1967).

As the GCs are binary, a subset of them will be active at any given time, depending on their inputs from the mossy fibers. (See Fig. 6 below for a visualization of a GC activity pattern over an epoch.) Thus, total PF activity (which is the same as GC activity) on any time-step is simply the number of GCs that are active. The InMin algorithm would also work with continuously valued GCs. The use of binary GCs simplifies the model without loss of generality.

The model distinguishes between PFs based on the identity of their underlying mossy fiber signal only for the purpose 
Table 1 The mossy fiber input signals to the cerebellum, along with their thresholds, number of phases, and number of GCs per sign-phase combination

\begin{tabular}{llll}
\hline Signal $(x)$ & Threshold $\left(T_{x}\right)$ & $\begin{array}{l}\text { Number } \\
\text { of phases }\end{array}$ & Number of GCs $\left(n_{x}\right)$ \\
\hline $\mathbf{e}$ & 0.1 radians & 6 & 8 \\
$\dot{\mathbf{e}}$ & 0.1 radians/s & 6 & 8 \\
$\mathbf{r}$ & 0.125 radians & 6 & 16 \\
$\dot{\mathbf{r}}$ & 0.125 radians/s & 6 & 16 \\
$\dot{\mathbf{s}}$ & 17 radians/s & 1 & 16 \\
\hline
\end{tabular}

of routing the fibers to particular MZs. Within an MZ, all PFs are treated equally. The model does not attempt to apply any special interpretation to certain PFs based on their origin. In particular, the PFs carrying error and efference copy signals are indistinguishable from PFs carrying eye position and velocity signals.

The error signals are segregated by dimension (see Fig. 2), which implies that each $\mathrm{MZ}$ receive only a subset of the available PFs. In particular, out of the 1,216 PFs each MZ receives 800. Matrix $\mathbf{W}$ represents the synaptic connections between the PFs and PCs within an MZ, and the product $\mathbf{p}=\mathbf{W h}$ determines the outputs (in vector $\mathbf{p}$ ) of the PCs in the MZ. The vector of gain values $\mathbf{g}$ determines how much each PC contributes to the output $m$ of the MZ:

$m=\mathbf{g}^{\mathrm{T}} \mathbf{p}=\mathbf{g}^{\mathrm{T}} \mathbf{W h}$.

The cerebellar contribution $\mathbf{P}$ to the motoneuron command m (see Eq. 7) is then:

$\mathbf{P}=\left[\begin{array}{c}P_{\mathrm{h}} \\ P_{\mathrm{v}}\end{array}\right]=\left[\begin{array}{l}m_{\mathrm{r}}-m_{1} \\ m_{\mathrm{u}}-m_{\mathrm{d}}\end{array}\right]$,

where subscripts $\mathrm{h}$ and $\mathrm{v}$ designate horizontal and vertical, and $\mathrm{r}, 1, \mathrm{u}$, and d designate the right, left, up, and down MZs, respectively (see Fig. 2).

\subsection{Learning algorithm}

The ultimate objective of the InMin learning algorithm is to configure and combine the PC responses in such a way that the resulting regulation of pursuit by the model cerebellum is accurate and efficient. "Input Minimization" describes the direct objective of the algorithm: to minimize the activity over all the PFs (which originate from GCs) that project to the PCs in a given MZ. Roughly, half of this activity is due to the inputs that represent the state of the eye, while the other half is due to the inputs that represent retinal error and saccade command efference copy. While all these inputs are controllable by some combination of PC outputs, only changes that reduce the retinal error (and consequently reduce catchup saccades) will result in a net decrease in GC activity.
Therefore, minimizing total PF (GC) activity will minimize error and efference copy, and ensure that regulation by the model cerebellum results in pursuit that is both accurate (low retinal error) and efficient (few catch-up saccades).

The InMin algorithm is essentially a gradient descent using PF activity as the cost function. Thus, the key problem that the algorithm must solve is to detect the gradient with respect to the synaptic weights, which in this model are represented by the PF-to-PC weights $\mathbf{W}$ and the PC output weights g. Gradient descent in such a high-dimensional space would be very expensive. We apply self-organizing map (SOM) learning (Kohonen 1997) to $\mathbf{W}$, leaving only the gains $\mathbf{g}$ to be learned explicitly by gradient descent. The general method of gradient descent employed in InMin involves perturbation rather then explicit gradient computation (Alspector et al. 1993; Venkatesh 1993). The specific version, called "perturbative reinforcement learning", works by generating small random changes in $\mathbf{g}$ and measuring their effects on overall $\mathrm{PF}$ activity, which serves as a negative reinforcement signal. Both learning methods run concurrently during the entire training period.

Initially, $\mathbf{W}$ contains random positive values. Each row of $\mathbf{W}$ is associated with an individual $\mathrm{PC}$ in an $\mathrm{MZ}$ and is normalized to 1 :

$\left\|\mathbf{W}_{\text {row i }}\right\|=1, \quad i=1, \ldots, n_{\mathrm{pc}}$,

where $n_{\mathrm{pc}}=12$ is the number of PCs in each MZ. The PC output weights in vector $\mathbf{g}$ are all initially zero.

All long-term weight changes are initiated by CF spikes. By definition, there is one CF per MZ. The model presented here contains four independently firing $\mathrm{CF}$ s associated with the four MZs (Fig. 2). The CF spikes generated by the model inferior olive are random. Following (Kuroda et al. 2001), the CF projecting to a given MZ spikes at a given time-step if:

$B_{c} \Delta t>u$,

where $B_{\mathrm{c}}=1 \mathrm{spike} / \mathrm{s}$ is the base firing rate and $u$ is a random number in $[0,1]$ that is drawn at each iteration from a uniform distribution. Apart from a refractory period of $50 \mathrm{~ms}$, the CF firing probability is independent of any previous spike. The resulting pattern of spike timing is discussed below.

When a CF spike occurs, it triggers both SOM and perturbative learning. The following two subsections describe these in detail. During the period between CF spikes, the algorithm measures smoothed PF activity to detect its trend, which is used as a negative reinforcement signal for perturbative learning. In the Marr/Albus paradigm, CF spikes carry explicit error signals and thus play an entirely different role from the one described for InMin. For the purpose of comparison, we also describe a delta-rule learning setup that uses an explicit error signal carried by the CFs, as in Marr/Albus. 


\subsubsection{Unsupervised (SOM) learning}

Self-organizing map learning generally proceeds by first selecting the unit in the network whose weight vector is closest (by some measure, generally the inner-product) to the input vector (Kohonen 1997). In the model, a CF spike initiates a competition between the PCs, implemented simply using a max operation. The most active PC is the one whose synaptic weights most closely match the pattern on the PFs at the time of the CF spike:

$j=\underset{i}{\arg \max } p_{i}$,

where $p_{i}=\mathbf{W}_{\text {row }} \mathbf{h}$ is the activity of a given $\mathrm{PC}$ in $\mathbf{p}$, and $j$ is the index of the most active PC. The next step in the SOM algorithm is for the winner and its four nearest neighbors (two neighbors on either side) to update their weights from the PFs:

$\mathbf{W}_{\text {row } i} \leftarrow \frac{\mathbf{W}_{\text {row } i}+2^{-|j-i|} L_{c} \mathbf{h}^{\mathrm{T}}}{\left\|\mathbf{W}_{\text {row } i}+2^{-|j-i|} L_{c} \mathbf{h}^{\mathrm{T}}\right\|}, \quad i=j-2, \ldots, j+2$,

where $L_{c}=0.0003$ is the SOM learning rate. Note that the learning rate is applied to the weight vector of the winner at full strength, but to its neighbors at geometrically decreasing strength. To be consistent with the likely topology of a real MZ, the neighborhood relationship does not connect PC number $n_{\mathrm{pc}}$ and PC number 1 (i.e., PCs in an MZ are not arranged in a closed circle). The effect of this learning is the emergence of PCs that are specialized for specific PF input patterns (see Sect. 3). These PC responses act as basis functions which are combined according to the gains $\mathbf{g}$ to form the output waveform of the MZ (see Eq.9).

\subsubsection{Perturbative learning}

There is some (not necessarily unique) combination of the PC outputs that will generate the signal needed to reduce retinal error in the simulated pursuit system. The objective of perturbative learning is to determine the output weights $\mathbf{g}$ for the given MZ. In order to implement perturbative learning, each $\mathrm{CF}$ spike induces a small and temporary change $\Delta \mathbf{g}$ in the PC output weights (gains) of a given MZ. The vector of perturbations $\Delta \mathbf{g}$ has both positive and negative elements that are drawn from a uniform distribution. The size of the perturbations, as reflected by $\|\Delta \mathbf{g}\|$, is set dynamically as described below. The output of the $\mathrm{MZ}$ then becomes

$m=(\mathbf{g}+\Delta \mathbf{g})^{\mathrm{T}} \mathbf{p}$.

When the next CF spike arrives, the MZ evaluates whether the average PF activity is rising or falling. If it is falling fast enough, then some portion of $\Delta \mathbf{g}$ becomes permanent: $\mathbf{g} \leftarrow \mathbf{g}+L_{p} \Delta \mathbf{g}$

where $L_{p}=0.3$ is the perturbative learning rate. This process then repeats with new values for the vector $\Delta \mathbf{g}$.

The expectation is that a change in $\mathrm{MZ}$ output should have an affect on pursuit performance that results in a change in the PF activity reaching the MZ. The aim is to minimize the controllable portion of the PF input (i.e., the portion of the input that can be affected by the cerebellar contribution to pursuit). Measuring PF activity is therefore a key part of the model. Total PF activity can fluctuate up or down regardless of variation in the controllable portion of the input. However, a larger than expected fluctuation up or down indicates motion toward a worse or better configuration, respectively, of the weights in $\mathbf{g}$.

The model measures the rate of change in PF activity by comparing the outputs of two leaky integrators:

$\bar{h}_{1} \leftarrow \gamma_{1} \bar{h}_{1}+\left(1-\gamma_{1}\right)|\mathbf{h}|$ and

$\bar{h}_{\mathrm{s}} \leftarrow \gamma_{\mathrm{s}} \bar{h}_{\mathrm{s}}+\left(1-\gamma_{\mathrm{s}}\right)|\mathbf{h}|$,

where the subscripts $\mathrm{s}$ and 1 refer to short and long term, respectively, $\bar{h}$ is smoothed PF activity, $\gamma$ is the decay constant of a leaky integrator, and $|\cdot|$ is the vector 1 -norm. The simulations reported here use $\gamma_{1}=0.9980$ and $\gamma_{\mathrm{s}}=0.9967$. Effectively, the time constant of $\bar{h}_{\mathrm{s}}$ is $3 \mathrm{~s}$ (1 epoch), and the time constant of $\bar{h}_{1}$ is $5 \mathrm{~s}$ (almost 2 epochs). These time constants are arbitrary values chosen empirically to give good results. The difference between the outputs of the two integrators reflects the rate of change in average PF activity:

$\Delta \bar{h}=\bar{h}_{\mathrm{s}}-\bar{h}_{1}$.

The model sets the threshold for the expected amount of fluctuation in $\Delta \bar{h}$ by noting its maximum and minimum values in the previous epoch:

$T_{\Delta}=\frac{\min \Delta \bar{h}-\max \Delta \bar{h}}{2}$.

Note that $T_{\Delta}$ is always a negative number. Any time $\Delta \bar{h}$ falls below $T_{\Delta}$, an improvement has occurred, and the model updates the weights $\mathbf{g}$ according to Eq. 16.

The size of the perturbation $\|\Delta \mathbf{g}\|$ must be such that perturbations in the adaptive direction are sufficient to drive the change in PF activity $\Delta \bar{h}$ below the change threshold $T_{\Delta}$. At the same time, perturbation size should be relatively small to avoid destabilizing the system. The model chooses the perturbation size based on the current value of the change threshold $T_{\Delta}$ :

$\|\Delta \mathbf{g}\|=-0.125 A T_{\Delta}$,

where $A$ is the amplitude of object motion (see Eq. 1 and 2 ), and 0.125 is an arbitrary constant determined empirically to give good results. In addition to Eq.20, placing a hard upper limit on $\Delta \mathbf{g}$ and applying a non-linear ramp can help 
the simulation converge better. While individual elements of the perturbation vector $\Delta \mathbf{g}$ can be positive or negative, the PC output weight values in vector $\mathbf{g}$ are constrained to be non-negative.

Self-organizing map and perturbative training occur concurrently throughout the training period, which continues until the error has been reduced below the criterion error. The criterion error is defined as the maximum distance between simulated eye position and target position at any time during a given epoch (infinity norm). The training for H3V2 (pretzel) patterns stops when the criterion error is less than $1 / 12$ th of the object motion amplitude $A$ (see Eq. 1 and 2) or about 0.0083 radians. For H2V2 (circular) patterns the error must be less than $1 / 15$ th of $A$, or about 0.0066 radians. The tighter tolerance on the circle is needed to match the performance of InMin on this problem to that achievable using the Marr/ Albus paradigm. Comparison of the responses of simulated PCs following training under the two paradigms forms the basis of an experimentally feasible test that can distinguish between InMin and Marr/Albus in cerebellar learning (see Sects. 3 and 4).

While the unsupervised (SOM) component of learning is the same in this version of InMin as in the original version (Anastasio 2001a), the perturbative component is entirely different. The main improvement has been to enable the algorithm to measure smoothed error (and efference copy) signals over many time-steps rather than snap-shots of error at single time steps. The improvement enables the algorithm to be used for a wider variety of learning problems. A more detailed comparison between the current and original versions of InMin is made later (see Sect. 4).

As a result of SOM learning, each $\mathrm{PC}$ in the model becomes specialized for its own preferred input (PF) pattern, which occurs at a specific time point during a pursuit task. As a result of perturbative learning, each PC adjusts its contribution to the ongoing pursuit behavior (output gain) according to its output over the full cycle. Thus, the effect of each PC on pursuit is a function both of its output gain and the correlation between its PF input weights and the current PF input pattern. InMin training causes the PCs to act as feedforward pattern correlators (Anastasio 2001b), and in this respect the model is consistent with the Marr/Albus paradigm. Although the behavior resulting from training is similar, the training procedure in InMin, which is a combination of unsupervised and reinforcement learning, is drastically different from Marr/Albus training, which involves supervised learning using the delta rule.

\subsubsection{Delta-rule learning}

In order to generate predictions about the behavior of InMin compared with the Marr/Albus paradigm, the model also includes a simple delta-rule learning mode (Widrow and
Hoff 1960). This mode completely replaces the unsupervised (SOM) and perturbative reinforcement learning procedures with the weight update

$$
\mathbf{W}_{\text {row } i} \leftarrow \mathbf{M}_{\text {row } i} \circ\left(\mathbf{W}_{\text {row } i}+L_{\mathrm{d}} \dot{\mathbf{r}}^{\mathrm{T}} \dot{\mathbf{x}} \mathbf{h}^{\mathrm{T}}\right), \quad i=1, \ldots, n_{\mathrm{pc}}
$$

where $\mathbf{M}$ is a Boolean masking matrix of the same form as W that indicates which PF-to-PC synapses are connected, o is element-wise multiplication, $L_{\mathrm{d}}$ is the delta-rule learning rate, and $\dot{\mathbf{x}}$ is a normalized vector in the direction (up, down, left, or right, see Fig. 2) in which the given MZ is sensitive to retinal slip error $\dot{\mathbf{r}}$. In the Marr/Albus paradigm, the signal $\dot{\mathbf{r}}^{\mathrm{T}} \dot{\mathbf{x}}$ would be carried by the CFs. The purpose of the masking matrix $\mathbf{M}$ is to model an $\mathrm{MZ}$ where each $\mathrm{PF}$ is connected to only a subset of the PCs and to study what effect this has on delta-rule learning, if any (see Sect. 3). $\mathbf{M}$ is a binary matrix in which each element takes value 1 with a specified probability and is 0 otherwise. Unlike SOM and perturbative learning, which occur only after CF spikes, the update in Eq. 21 occurs at every time-step. For simplicity, the PC output weights (gains) are initially equal and remain constant at:

$g_{i}=\frac{0.2}{n_{\mathrm{pc}}}, \quad i=1, \ldots, n_{\mathrm{pc}}$.

\subsection{Analysis procedures}

Most of the results reported here are simply plots of simulation variables against time (discrete time steps), such as PC responses, or of simulation variables against each other, such as horizontal versus vertical object or eye position. Somewhat more specialized analysis procedures include the construction of the CF spike histogram and the determination of PC response vectors.

\subsubsection{CF spike timing histogram}

This is a histogram of the number of CF spikes that occur in each of a range of time bins after a trigger $\mathrm{CF}$ spike, with every CF spike functioning as a trigger event for subsequent $\mathrm{CF}$ spikes. The $\mathrm{CF}$ spike trace $c(i)$ for a given run of the model is defined as

$c(i)= \begin{cases}1, & \text { if a spike occurred at step } i, \\ 0, & \text { otherwise }\end{cases}$

where $i$ is an integer in $(-\infty, \infty)$ that indexes the discrete time-steps in the simulation. Let $n_{\text {steps }}=300$ be the number of time-steps in one epoch, and let $n_{\text {trace }}$ be the total number of time-steps in a given simulation, which includes all of its epochs. The function $c(i)$ has non-zero entries for $i$ only in $\left[1, n_{\text {trace }}\right]$.

The process can be thought of as a moving window that slides over the CF spike trace. Whenever a CF spike occurs, 
it sets the start point for the window, and all subsequent $\mathrm{CF}$ spikes increment histogram bins at their respective positions in the window. This repeats for all CF spikes, including those that have contributed to bins under one or more window positions. The value in each bin of the histogram is computed as:

$$
\begin{aligned}
\mathrm{H}(b) & =\sum_{i=1}^{n_{\text {trace }}}\left\{\begin{array}{l}
1, \text { if spike at step } i \text { AND spike } b \text { steps later } \\
0, \text { otherwise }
\end{array}\right. \\
& =\sum_{i=1}^{n_{\text {trace }}} c(i) c(i+b)
\end{aligned}
$$

where $b$ is the bin number. Equation 24 is the formula actually used to compute the CF spike histogram. However, since $c$ is always zero outside of $\left[1, n_{\text {trace }}\right]$, this is equivalent to

$\mathrm{H}(b)=\sum_{i=-\infty}^{\infty} c(i) c(i+b)$

which is the discrete auto-correlation of the function $c$. The histogram computed according to Eq. 24 can be interpreted as though computed according to Eq. 25, and, in particular, a flat histogram would correspond to a uniform distribution of CF spike times or, equivalently, a lack of synchronicity between CF spikes. Finally, note that we restrict the domain of $b$ to $\left[1, n_{\text {steps }}\right]$ for simplicity of presentation.

\subsubsection{PC response vector determination}

This analysis procedure is adapted from Suh et al. (2000). The output of a PC can be approximated as a function of the position and velocity of the eye at a given point in time:

$p_{i}(t)=\dot{\mathbf{x}}_{i}^{\mathrm{T}} \dot{\mathbf{e}}(t)+\mathbf{x}_{i}^{\mathrm{T}} \mathbf{e}(t)+\beta_{i}$,

where $\dot{\mathbf{x}}_{i}$ and $\mathbf{x}_{i}$ are the vectors of coefficients, specific to each PC $i$, relating the horizontal and vertical components of eye velocity and position, respectively, to the response $p_{i}$ of the PC, and $\beta_{i}$ is a constant. Given traces at each discrete time-step $[1, n]$ of $p_{i}$, eye velocity $\dot{\mathbf{e}}$, and eye position $\mathbf{e}$, we can assemble an over-determined system of simultaneous equations

$$
\left[\begin{array}{lll}
\dot{\mathbf{e}}^{\mathrm{T}}(1) & \mathbf{e}^{\mathrm{T}}(1) & 1 \\
\dot{\mathbf{e}}^{\mathrm{T}}(2) & \mathbf{e}^{\mathrm{T}}(2) & 1 \\
\vdots & \vdots & \vdots \\
\dot{\mathbf{e}}^{\mathrm{T}}(n) & \mathbf{e}^{\mathrm{T}}(n) & 1
\end{array}\right]\left[\begin{array}{l}
\dot{\mathbf{x}}_{i} \\
\mathbf{x}_{i} \\
\beta_{i}
\end{array}\right]=\left[\begin{array}{l}
p_{i}(1) \\
p_{i}(2) \\
\vdots \\
p_{i}(n)
\end{array}\right]
$$

and solve them for the parameters $\dot{\mathbf{x}}_{i}, \mathbf{x}_{i}$, and $\beta_{i}$ using a linear least squares method. The vectors $\dot{\mathbf{x}}_{i}$ and $\mathbf{x}_{i}$ can be viewed as sensitivities to horizontal and vertical eye velocity and position, while $\beta_{i}$ is the baseline activity.

\section{Results}

The aim of the InMin algorithm in the context of this model of smooth pursuit is to train the cerebellar component to overcome the limitations of the cortical components and produce pursuit that is both accurate and smooth (i.e., devoid of catchup saccades). The cortical components (see Sects. 1 and 2) are simple models of the smooth (FEFsem) and saccadic (FEFsac) sub-regions of the frontal eye fields. The FEFsem component functions as a velocity servomechanism that minimizes retinal slip error. The gain of the FEFsem component is limited to prevent instability due to the delay inherent in the retinal slip signal (Milsum 1966). The FEFsac component functions as a sampled data system that transiently reduces retinal position error whenever it exceeds a threshold (ibid). The FEFsem and FEFsac components work together, respectively, to reduce velocity and position error but, due to their unavoidable limitations, the pursuit they produce is too weak and too choppy. By functioning as a feedforward controller, the cerebellar component learns to provide a predictive and high-gain command that essentially takes over from FEFsem and FEFsac during pursuit. The cerebellar component does not completely obviate the cortical components because it operates only for object motions with which the cerebellum has had experience sufficient for learning.

The InMin algorithm is capable of training the cerebellar component of the model by minimizing the overall amount of input received by PCs from GCs via their PFs. Nearly half of this input encodes the position or velocity of the eye, while slightly more than half encodes error and effort. Decreases in the amplitude of the eye trajectory will be offset by increases in error and effort unless the change improves pursuit performance. Similarly, increases in the amplitude of the eye trajectory will be discouraged unless there is a corresponding decrease in error and effort. Thus, InMin produces adaptive changes in the performance of the cerebellar component of the model by causing it to minimize the total amount of PF input it receives.

Learning and performance in the model are studied using two distinct object trajectories (Kettner et al. 1996). Both are two-dimensional (horizontal and vertical) and both are periodic with the same base frequency of $1 / 3 \mathrm{~Hz}$. The H3V2 (pretzel) pattern has horizontal and vertical components at 3 and 2 times the base frequency, respectively, while the H2V2 (circle) has horizontal and vertical components both at 2 times the base frequency. The results reported below demonstrate the ability of the InMin algorithm to train the cerebellar component of the model to achieve good performance on these trajectories. They also compare the responses of PCs in the trained model with data on real PCs and use the model to derive a testable prediction that should distinguish between learning mechanisms like InMin and Marr/Albus in actual cerebellar plasticity. 

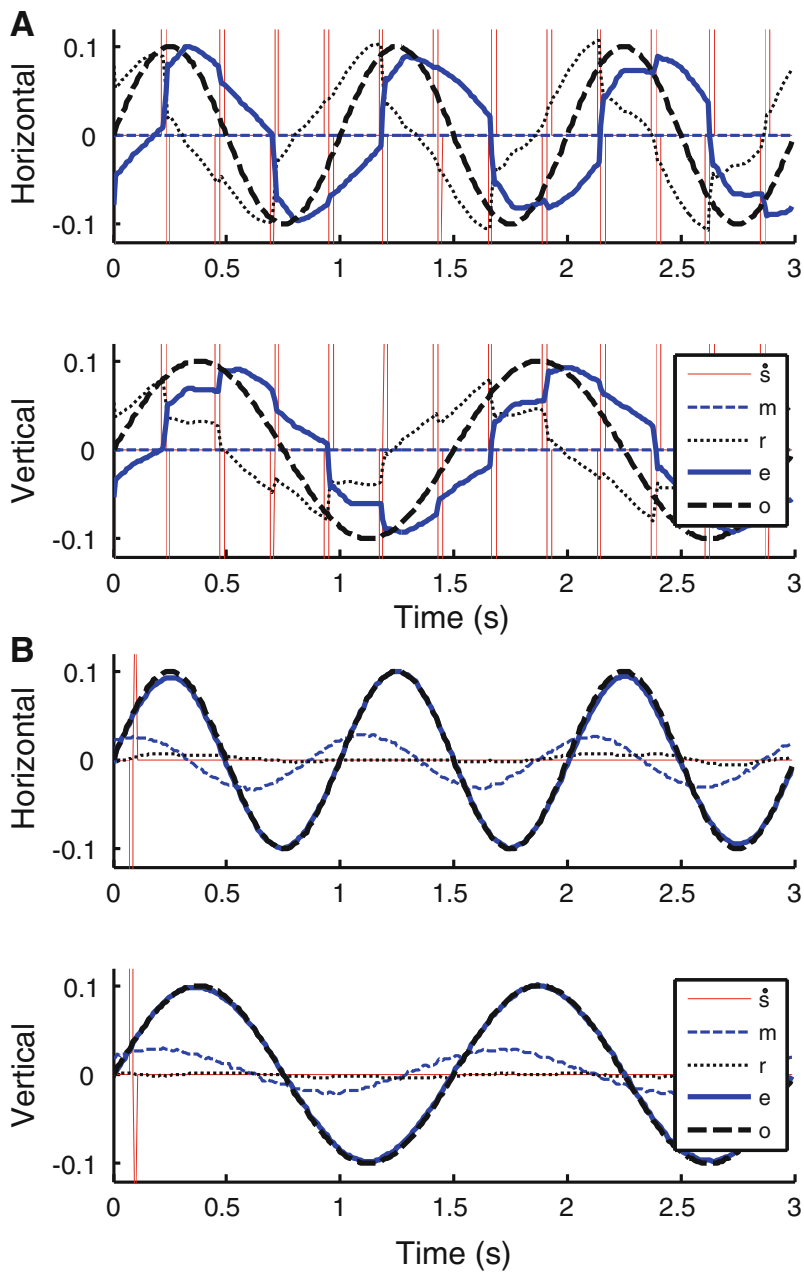

Fig. 4 Plots of key signals in the model during H3V2 (pretzel) pursuit. The vertical and horizontal components appear separately. A Start of training. B After InMin training to criterion. The five variables illustrated are: saccade command $(\dot{s})$, net command from each cerebellar microzone $(m)$, retinal position error $(r)$, eye position $(e)$ and object position $(o)$ in the dimension corresponding to each plot

\subsection{Cerebellar pursuit}

An overview of cerebellar learning in this model is provided in Fig. 4, which shows the horizontal and vertical components separately as functions of time. Figure 4A (top, horizontal; bottom, vertical) shows the performance of the model immediately after it is first presented with the H3V2 (pretzel) object motion pattern and before any appreciable cerebellar learning has taken place. At this point, there is very little output from the cerebellar component (thin dashed lines). Practically all of the pursuit command comes from the smooth eye movement and saccadic components of the model FEF. Eye position (thick solid lines) is a very rough sinusoid, with an underlying smooth undulation produced by the FEFsem component (not shown) that is augmented quasi-periodically by saccades produced by FEFsac saccadic commands (truncated vertical lines), which appear as pairs of sharp pulses with opposite signs that typically exceed the vertical scale of the plot. This very roughly sinusoidal eye position trace (thick solid line) has approximately the same amplitude as the object position trace (thick dashed lines), but exhibits a notable phase lag. Retinal position error (dotted lines) and slip velocity error (not shown) cycle with the movement of the object and exhibit large amplitudes.

Figure 4B (top, horizontal; bottom, vertical) shows the performance of the model after simulated cerebellar adaptation. Saccades and retinal position error are minimal. At this point, the cerebellar output is smoothly sinusoidal and is sufficiently phase-advanced relative to object position to compensate for input delays and the mechanical lag of the eye plant.

The characteristic "pretzel" profile of the H3V2 trajectory is illustrated in Fig. 5, in which horizontal and vertical object positions are plotted against each other (dashed line). The figure also plots eye position after training (solid line) and provides a qualitative view of how well the final output tracks the moving object. Training initially produces large decreases in error, after which it brings error toward criterion more gradually. This example took 90,378 epochs to reach criterion, with a final error of 0.0076 radians. At $3 \mathrm{~s}$ per epoch, this is equivalent to about $75 \mathrm{~h}$ and $19 \mathrm{~min}$ of training time. Figures 4 and 5 illustrate the result that the InMin algorithm is capable of simulating adaptive motor learning in the pursuit context. This learning occurs in the complete absence of error signals carried by CFs.

\subsection{Parallel and climbing fiber activity}

In the InMin algorithm, CF spikes serve as "learn now" signals that initiate adaptive processes in each MZ (see

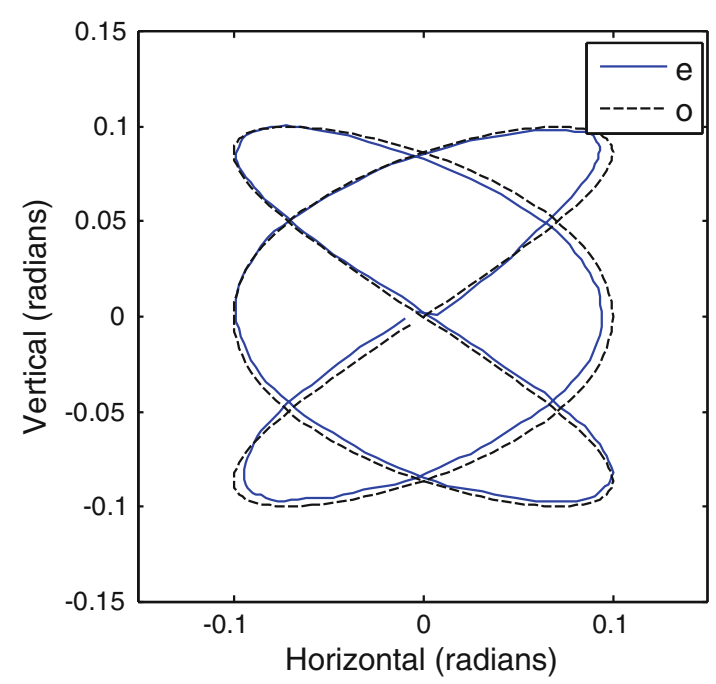

Fig. 5 Two-dimensional plot of object position for the H3V2 (pretzel) pattern (dashed), along with simulated eye position trajectory produced by the model after training (solid) 
Fig. 6 Output of model granule cells (GCs) afferent to the "right" MZ during H3V2 pursuit. The GCs give rise to the parallel fibers (PFs) in the model. A Start of training. B After InMin training to criterion. Note that the activities of the error and efference copy related PFs (granule cells 1 through 416) have been minimized as a result of training. The vertical rule in plot B marks a specific pattern of PF activity (see text)

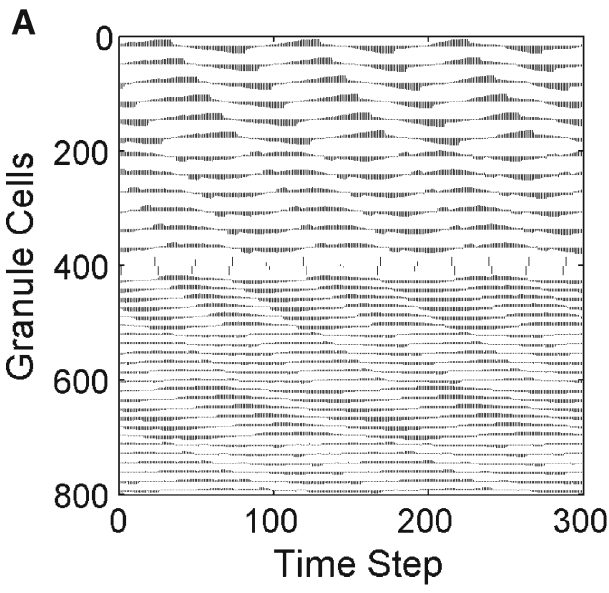

Sect.2). Signals related to eye motion, retinal error, and saccadic efference copy are sent to the model PCs over the mossy-fiber/granule-cell/parallel-fiber pathway. The 800 binary GCs produce an expansive re-coding of the relevant subset of the mossy fiber signals. Almost half of the PFs carry signals related to eye motion. Specifically, they encode either horizontal or vertical eye movement at 12 different phases. The remaining PFs carry horizontal or vertical retinal error at 12 different phases or saccade command velocity efference copy. PF activity is illustrated in Fig. 6.

The set of $800 \mathrm{PFs}$ that impinge on each MZ of the model cerebellum can be roughly divided into two subsets. The first subset encodes retinal error and saccadic efference copy, and the second subset encodes horizontal and vertical eye motions. Figure 6A shows a trace of PF activity before training (for the epoch depicted in Fig. 4A), while Fig. 6B shows a trace of PF activity after training (for the epoch depicted in Fig. 4B). Note that the PF activity patterns in the second subset are smoother after training. The PF activity in the first subset, encoding retinal error and saccadic efference copy, is reduced to essentially zero by the end of the training. The PFs carrying retinal error and saccadic efference copy signals are not explicitly labeled as such. The InMin algorithm is able to eliminate almost all of the error and saccadic efference copy activity by minimizing overall PF activity.

The use of PFs to carry error signals is a departure from the Marr/Albus paradigm, in which CFs carry explicit error signals (see Sect.1). Figure 7 shows the histogram of CF spikes for the training illustrated in Figs. 4 through 6. This histogram is essentially flat, showing that the distribution of intervals between CF spikes in the model is uniformly random, by design (see Sect. 2). The CFs in the model are not modulated by retinal position or velocity error, or by any other quantity related directly to pursuit. As the retinal error signals cycle in synchrony with object motion, any correlation between them and CF spikes would show up as peaks in the histogram. Other than the $50 \mathrm{~ms}$ refractory period, there

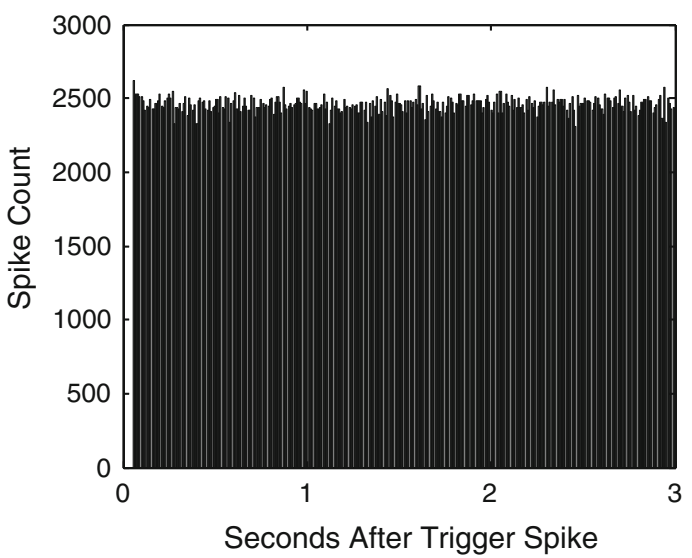

Fig. 7 Spike timing histogram for the climbing fiber impinging on the "right" MZ during training on the H3V2 (pretzel) pattern. The spike-count distribution is uniform except for the $\mathrm{CF}$ refractory period of $50 \mathrm{~ms}$. Spike timing histograms for the climbing fibers impinging on the other three MZs are similar

is no significant timing relationship between CF spikes. This verifies that in our model $\mathrm{CF}$ spikes carry no information other than a randomly timed "learn now" signal.

\subsection{Purkinje cell responses}

Early in InMin training, unsupervised SOM causes the model PCs to become specialized for specific patterns of PF input. These patterns correspond to a specific phase of the eye trajectory. Initially, the PF-to-PC weights $\mathbf{W}$ are random, and each PC will only slightly favor a particular part of the combined PF input. Figure 8 shows the responses of all 12 PCs in the MZ controlling rightward eye movement following training (the responses of PCs in the other MZs are qualitatively similar). The SOM training stage causes each PC to become specialized for a different temporal segment of the PF input, and it roughly divides the input epoch so that each temporal segment of it has a PC that is specialized for it. (This is even more apparent for circular pursuit, see next subsection.) Each $\mathrm{PC}$ response is a mixture of the horizontal and vertical eye 
Fig. 8 Outputs of all 12 PCs in the "right" MZ before weighting by g. The PC responses in the other three MZs are qualitatively similar
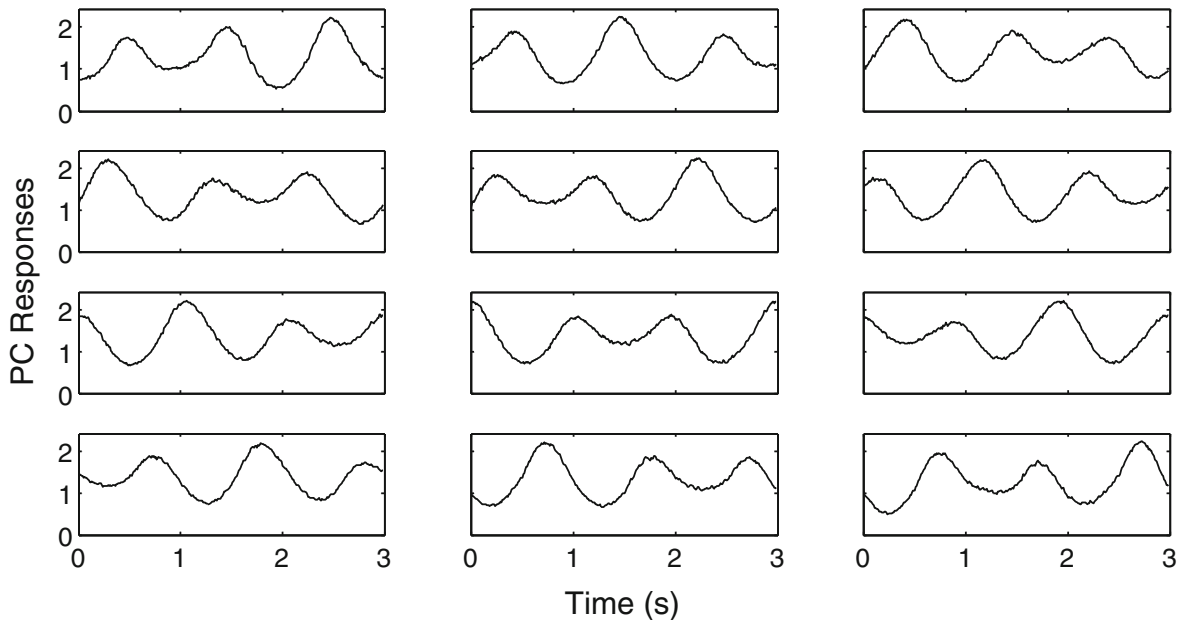

movement signals with some phase shift between them. The response waveforms resemble those of real monkey PCs following training to pursue the H3V2 (pretzel) trajectory (Suh et al. 2000).

The PC responses can be thought of as a set of basis functions that can be combined linearly to reproduce the desired output function (a pursuit command in this case). Although the $\mathrm{PC}$ responses are essentially motor commands, they can also be viewed as having "sensitivity" to some combination of horizontal and vertical eye position and velocity (Suh et al. 2000; Leung et al. 2000). Figure 9 shows an analysis of the response waveforms for all $48 \mathrm{PCs}$ using a least squares method to find the coefficients that relate the $\mathrm{PC}$ response to horizontal and vertical eye position and velocity (plus a constant, see Sect. 2). The root-mean-squared error over all $48 \mathrm{PCs}$ is 0.0573 . Since the PC outputs are scaled to be near 1 , the fitting functions account for at least $94 \%$ of the PC responses on average.

The results can be viewed in two dimensions as velocity (Fig. 9A) and position (Fig.9B) sensitivity (or motor command) vectors. The wide dispersal in all directions is similar to that observed experimentally (Suh et al. 2000). One might naively suppose that a model PC with its velocity sensitivity in a particular direction would have its position sensitivity in the same direction, but this is not the case. A plot of the angles of the velocity against the position sensitivity vectors for all $48 \mathrm{PCs}$ is shown in Fig. 9C. If the directions of velocity and position sensitivity were the same, then all of the points would fall along the diagonal line. Instead, the points are spread uniformly over the plot. The variability in velocity versus position angles is similar to that observed experimentally (Suh et al. 2000).

This behavior is simulated by the model because the SOM stage of InMin training causes each PC to become specialized for its own specific temporal segment of the PF input. One such segment, corresponding to a specific temporal pattern of PF input, is indicated by the vertical line, roughly at time-step 240, in Fig. 6B. A PC specialized for this pattern would respond maximally for horizontal position (the first PF sinusoidal bundle in the second subset) but for vertical velocity (the 19th PF sinusoidal bundle in the second subset). Each temporal segment has its own unique combination of direction and phase, and this accounts for the dispersion in velocity versus position angles shown in Fig. 9C.

\subsection{Predictive behavior}

The output of the cerebellar component of the model is by nature predictive. It learns to anticipate the required motor command based on the current state of the eye (and any remaining retinal error). Kettner and coworkers studied the predictive nature of the pursuit in the monkey (Leung and Kettner 1997; Suh et al. 2000). They devised a pursuit task in which the trajectory of the target sometimes changes in a way that cannot be predicted based on previous training, to elucidate what parts of the pursuit eye movement are generated by learned predictive control and what parts are generated by other control paths. The learned pattern is a simple circular trajectory, and the perturbation is a removal of the horizontal component of object motion for $1 / 2$ cycle resulting in a vertical traverse across the diameter of the circle. Figure 10 shows a reproduction of this task for our model. No learning occurs during the perturbation. The circular object motion trajectory is $\mathrm{H} 2 \mathrm{~V} 2$. It took 6,716 epochs to reach criterion on the circular trajectory without the perturbation. This represents about $5 \mathrm{~h} 36 \mathrm{~min}$ of simulated learning time. The final error was 0.0066 radians.

Monkeys (Leung and Kettner 1997), the model of Kettner and coworkers (Kettner et al. 1997), and our model all have similar behavior at the start of the surprise motion: they continue on the original circular trajectory for at least the visual system delay of $100 \mathrm{~ms}$. After that, the smooth pursuit control pathways steer the trajectory away from the circle toward the true path along the diameter. Near this time a catch-up 

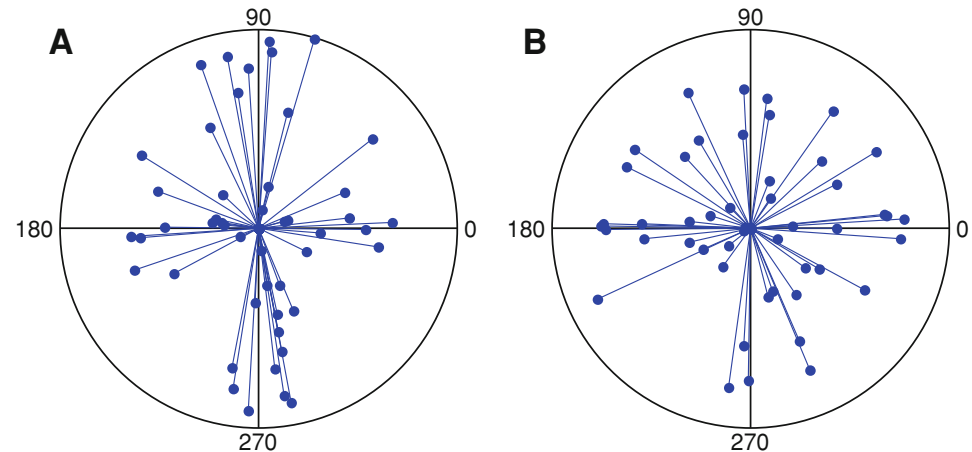

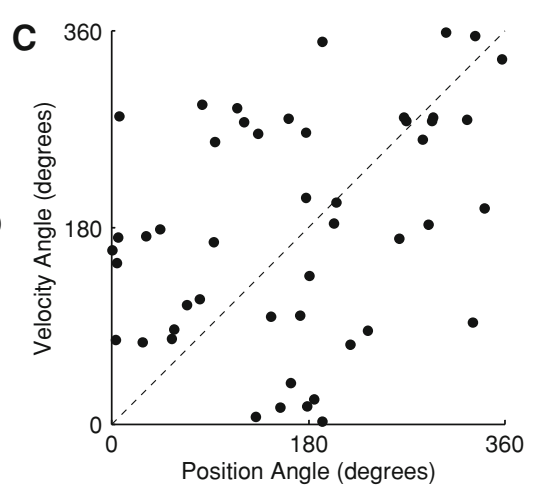

Fig. 9 Sensitivity vectors for PCs trained on the H3V2 (pretzel) pattern. The plots include all 48 PCs, 12 from each of the 4 MZs. A Velocity sensitivity vectors, which have been scaled down by $2 \pi f$ (with $f=3$ cycles/epoch $\times 1 / 3$ epoch/second $=1 \mathrm{~Hz}$ ) to bring their lengths into

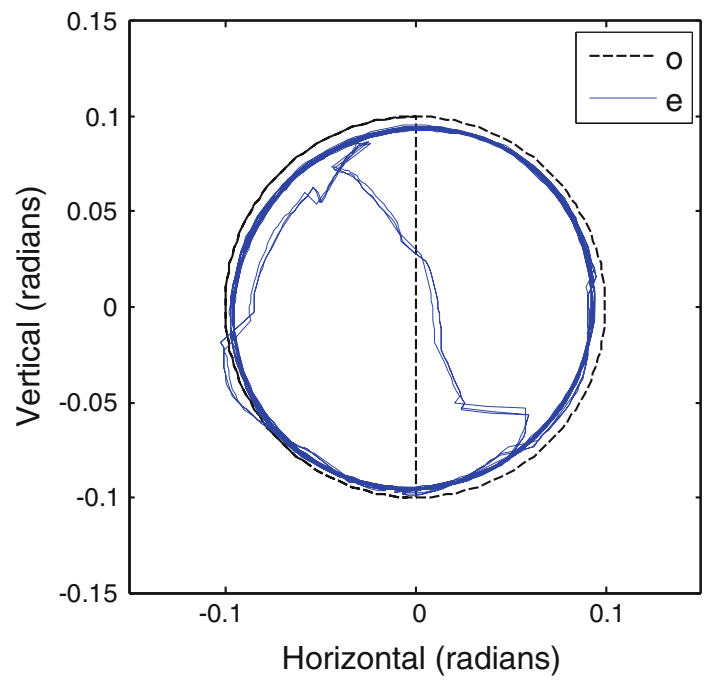

Fig. 10 Circular pursuit as simulated by the model following InMin training. Object position trajectory (dashed line) is plotted against simulated eye position trajectory (fine solid line). The perturbation (removal of the horizontal component of object motion for $1 / 2$ cycle) occurs after training and further demonstrates the predictive nature of the cerebellar contribution to simulated pursuit

saccade also occurs, which tends to put the eye close to the diameter. Real monkeys and both models follow the diameter and then veer toward the circular trajectory while the object is still moving upward on the diameter. Thus, the monkeys and both of the models all show horizontal pursuit eye movement before they could possibly be informed of horizontal object movement by visual input. This indicates the predictive capability of both the real and the simulated systems.

The trajectory of the eye in Fig. 10 as it moves up the vertical line is complex. Part of the reason for this is that, in our model, three different pathways are generating pursuit commands, each with their own unique characteristics. The the same range as the position vectors. B Position sensitivity vectors. C Velocity and position sensitivity vector angles plotted against each other for each PC

pulse from FEFsac is only $70 \%$ effective, so the eye does not fully reach the center line. FEFsem, on the other hand, continues to push the eye toward the center line based on input that is delayed by $100 \mathrm{~ms}$. As the eye nears the top of the circle, the predictive component from the cerebellum begins pushing the eye left. Finally, FEFsac issues another catch-up saccade command and the ensuing saccade puts the eye back on the circular trajectory, but not exactly in sync with the circular motion. This results in some additional adjustments for about $1 / 3$ of a cycle, mainly due to FEFsem and FEFsac, until the cerebellar component again recognizes its PF input and is fully re-engaged. The perturbation paradigm underscores the feedforward, correlative, and predictive nature of the cerebellar component of pursuit in the model. The behavior of the model on the perturbation task is qualitatively similar to that of monkeys (Leung and Kettner 1997).

\subsection{Comparing InMin and Marr/Albus}

Finally, we compare the effect of InMin training and Marr/ Albus delta-rule training on the PC responses in a MZ. InMin uses the SOM to train the PF-to-PC weights, so the outcome is a set of specialists for temporal segments of the PF input, as illustrated for the circular pattern in Fig. 11A. The variability in PC response phases is similar to that observed for real PCs following training on circular pursuit (Leung et al. 2000). In contrast, delta-rule training produces input connection weight changes according to the correlation between the performance error of, and the inputs to, a neural unit (see Eq.21). The delta rule trains the units to produce an output that minimizes the error. In the case of the Marr/ Albus paradigm applied in the context of smooth pursuit, PF-to-PC weight changes due to the delta rule would be based on the presumed retinal slip error signals transmitted to PCs over CFs. As all of the PCs in a MZ share the same CF, 
the delta rule will train all of those PCs to develop the same output response phase, which is specifically the phase of the output that minimizes retinal slip error (the error-minimizing output).

The model had the same configuration for delta-rule training as for InMin training. The only difference, in addition to delta-rule training of the PF-to-PC weights, was that the PC output weights were fixed under the Marr/Albus paradigm. As for InMin training, each MZ received all of the eye movement related $\mathrm{PFs}$, but received retinal error and saccadic efferency copy PFs that were segregated according to the dimension (horizontal or vertical) of the pursuit command they regulated (see Fig. 2). In order to explore the possible effects of differences in the PF input distribution, we modeled partial connectivity between the PCs and PFs of a given MZ (using the randomized masking matrix $\mathbf{M}$; see Eq. 21). We varied the PF connectivity between 100 and $10 \%$, with consistent results: in all cases, all of the PCs in an MZ developed the same output response phase. Figure 11B shows $\mathrm{PC}$ responses, and the overall performance of the cerebellar model, following delta-rule training under the Marr/Albus paradigm when the connectivity was $50 \%$.

The MZ outputs following training under the InMin and Marr/Albus paradigms are roughly the same (not shown), because both algorithms move them toward the errorminimizing waveform. While the PCs in an MZ following delta-rule training shared the same phase (that of the error-minimizing MZ output), independently of the percentage connectivity, the spread in response amplitude increased as connectivity decreased from 100 to $10 \%$. The general result that the PCs in an MZ share the same phase following
Marr/Albus training is also independent of the magnitude of the random starting values of the PF input weights, and of the fixed values of the PC output weights, and still occurs even if the fixed PC output weights take random values. These results can be used to derive an experimental test that would distinguish InMin from Marr/Albus in actual cerebellar plasticity (see Sect. 3).

\section{Discussion}

The InMin algorithm provides an alternative view of cerebellar learning in which the $\mathrm{CF}$ spikes initiate but do not direct synaptic weight changes, and error is reduced by minimizing overall PF activity. The behavior of the trained model is compatible with observations on smooth pursuit, and it offers new insight into the encoding of pursuit commands by PCs. An experimental test can be designed that would indicate whether the responses of real PCs after learning were more consistent with the InMin algorithm or with the traditional Marr/Albus paradigm, in which CFs carry explicit error signals that direct synaptic weight changes. These issues are considered below.

\subsection{Minimizing input minimizes both error and effort}

Models of cerebellar learning are generally preoccupied with the minimization of error, but movements made with a normal cerebellum are known for their efficiency as well as their accuracy (Gilman 1981). The cerebellum receives a huge amount of input from cortex, via the corticopontocerebellar
Fig. 11 Comparing simulated $\mathrm{PC}$ responses after training using InMin or Marr/Albus on circular pursuit. A Trained by InMin (same as Fig. 10, but without perturbation task). B Trained by delta-rule (Marr/Albus) with 50\% connectivity of PFs onto PCs, chosen at random. The heavy dashed curves mark one PC response for reference in both lower plots
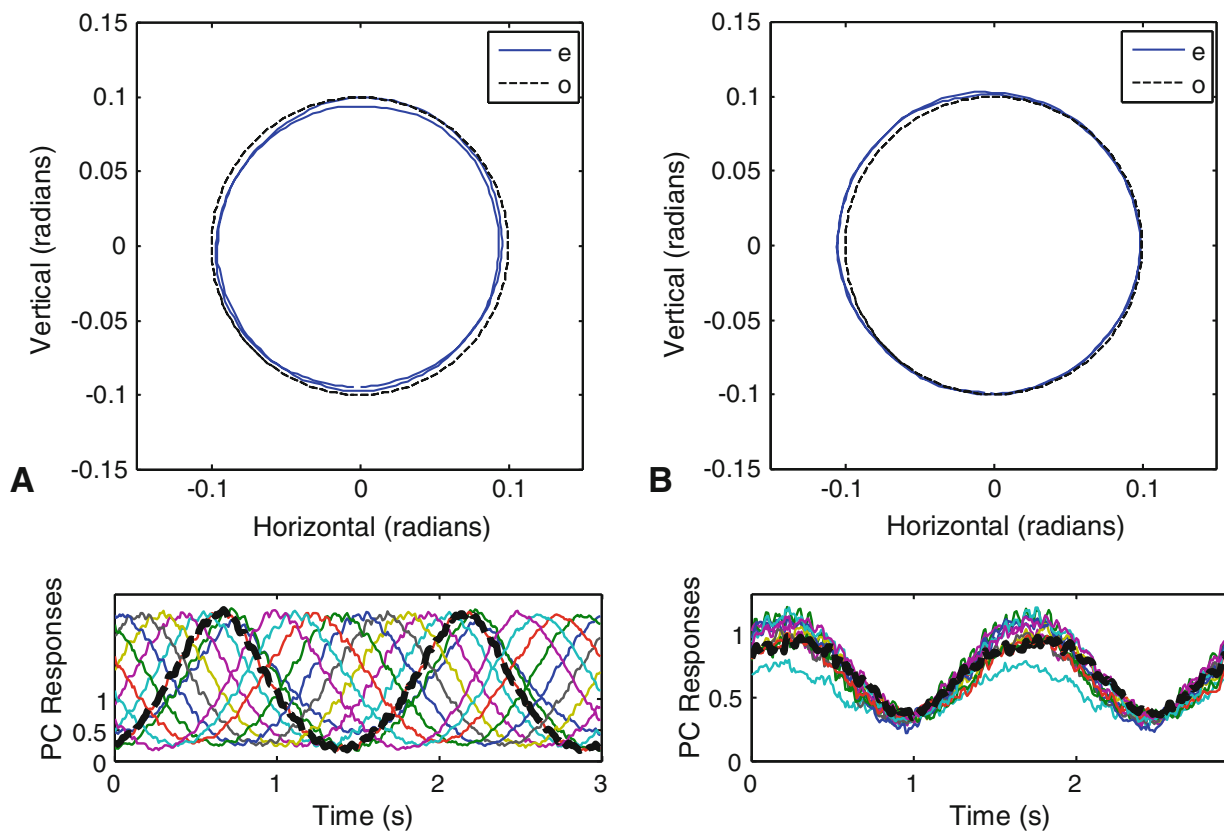

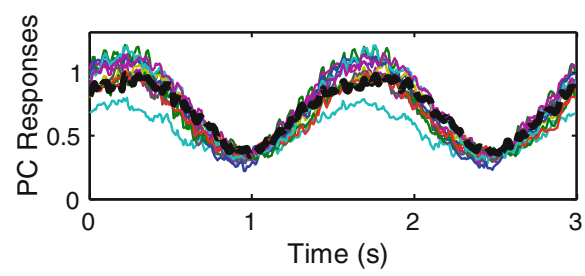


pathway (Butler and Hodos 1996; Nolte 2002). While much of this may provide the context in which specific cerebellar contributions should be made, and some may provide error signals, some of it may also provide efference copy of cortical movement commands, both voluntary and involuntary. Minimizing the overall amount of input to the cerebellum cannot change the context, but it can reduce both error and efference copy, thereby making movements simultaneously more accurate and more efficient.

The model of cerebellar control of pursuit by Kettner et al. (1997) included elements that generated catch-up saccade commands. These commands occurred less frequently as learning made pursuit more accurate, and so made catch-up saccades less necessary, but the commands themselves were not used in any way by the learning algorithm. By minimizing overall input, InMin uses both retinal slip error and catch-up saccade efference copy as (negative) reinforcement signals. The additional clues not only enhance learning, but also permit InMin simultaneously to optimize different aspects of system performance. By minimizing error, InMin reduces the activity of the direct servo loop, and by minimizing efference copy from the cortical saccadic system, InMin reduces the effort required to generate and carry out saccade commands. The pursuit model provides a simple illustration of the ability of the InMin algorithm to satisfy the dual goals of accuracy and efficiency.

\subsection{Comparison of the current and the initial versions of InMin}

The version of InMin used here to simulate cerebellar learning of predictive smooth pursuit was adapted from the initial version, which was used to simulate the cerebellar contribution to vestibulo-ocular reflex (VOR) adaptation (Anastasio 2001a). Both versions are similar in that they are organized in terms of cerebellar MZs, but the current, pursuit version operates in push-pull while the initial VOR version was unilateral. CFs spikes serve to initiate learning events and occur at a low, random rate in both versions.

Model PCs in both versions receive a large number of parallel fiber inputs from GCs (500-1000) that carry signals with a spread of phase relationships relative to pertinent oculomotor variables (e.g., eye or head motion). A subset of GCs in both versions carries error (retinal slip) signals that are delayed. In the pursuit version, some GCs also carry efference copy of catch-up saccade commands. Both versions use unsupervised learning to specialize model PCs for specific input patterns. Unsupervised learning in both cases involves a competition among model PCs at the time of occurrence of a CF spike to determine the winner (i.e., the cell having the largest response to the parallel fiber input), followed by cooperative training of the winner and its neighbors to bring their weight vectors closer to the current input vector.
Both versions of InMin use overall PF activity as a (negative) reinforcement signal, but they differ in the way they apply reinforcement learning to adjust the contributions of the model PCs to ongoing behavior.

In the initial version, only the output weight of the winning PC in a MZ is perturbed, while in the current version the output weights of all the PCs in a MZ are perturbed simultaneously. The initial version implements an eligibility trace, which essentially counts down the error feedback delay, and the effect of the perturbation on overall parallel fiber activity is determined immediately before and after the moment of eligibility. A significant decrease in parallel fiber activity after the moment of eligibility indicates that the perturbation has reduced the error, in which case the transient perturbation of the PC output weights is made permanent.

The current version does not implement an eligibility trace. It determines the effect of the perturbation on overall GC activity through comparison of two integrators, one leakier than the other. A significant decrease in GC activity in the more quickly as compared with the more slowly leaking integrator indicates that the perturbation has reduced the error, in which case part of the transient perturbation is made permanent. This difference in the two versions represents an improvement that enables the InMin algorithm to be applied in a wider variety of contexts. The challenges posed in the context of smooth pursuit illustrate the benefits of the new version.

The main difficulties in using InMin in the present context derive from performance-independent changes in error signals and unpredictability in efference copy signals. During pursuit of objects moving along cyclical trajectories, cyclical changes in error signals occur that are not directly tied to system performance (i.e., unless pursuit is completely errorfree, retinal slip error changes periodically with input signal amplitude). Also, efference copy from the saccadic component can occur at any point along the object trajectory.

The InMin learning mechanism relies, in part, on measuring the effect on total PC input of perturbing cerebellar parameters (e.g., the PC output weights). Perturbation size must be kept small to ensure stability of the InMin algorithm. Therefore, the performance-independent changes in error signals typically overwhelm any changes due to perturbation of cerebellar parameters. Also, the efference copies of saccade commands are brief, large, and relatively infrequent, so improvement only becomes apparent over a period of time. The model could be designed to record a trace of the error signal over an entire epoch and compare it with the trace in the next epoch, but such detailed and temporally specific memory is implausible. Instead, the deployment in the new version of a comparison of differentially leaky integrators enables detection of changes in a more plausible, more temporally limited manner. The neurobiological plausibility of the initial version of InMin has been discussed previously 
(Anastasio 2001a). That of the current version is discussed in the next subsection.

\subsection{Neurobiological plausibility of InMin}

A consensus has been building for the view that plasticity of the synapses between parallel fibers and PCs involves a combination of long-term depression (LTD) and long-term potentiation (LTP) (for reviews, see Hansel et al. 2001; Hartell 2002). LTD results in a decrease in the efficacy of parallel fiber synapses and is induced by conjunctive firing of PFs and CFs (Ito et al. 1982; Ito 2001). LTP is induced both pre- and post-synaptically by firing of parallel fibers in the absence of CF spikes (Hirano 1990; Lev-Ram et al. 2003; Sakurai 1987). These forms of cerebellar plasticity have been adapted to the Marr/Albus paradigm in recent models of cerebellar control of ocular following (Kuroda et al. 2001; Yamamoto et al. 2002). However, recent studies show that considerable cerebellar learning in oculomotor and other contexts is possible in the absence of LTD (van Alphen and De Zeeuw 2002; Welsh et al. 2005; Faulstich et al. 2006), leading to the conclusion that mechanisms other than LTD must also be at work in cerebellar learning (Hansel et al. 2001). InMin relies on several plastic mechanisms that are plausible in light of cerebellar findings.

For simplicity, unsupervised learning in InMin is implemented using the conventional SOM algorithm of Kohonen (1997). Thus, the unsupervised component of InMin involves competition within a $\mathrm{MZ}$, triggered by a CF spike, to determine which $\mathrm{PC}$ has the largest response to the pattern of $\mathrm{PF}$ input occurring at the time of the spike. The weights of active PFs onto the winning $\mathrm{PC}$ and its neighbors are strengthened, following which all the PF-to-PC weights of the winner and neighbors are normalized. The result of this training is that PCs become specialized for specific patterns of PF input. In addition, due to the map-forming properties of the SOM, model PCs that are neighbors in the network respond to similar patterns of PF input. Since similar patterns of PF input encode the sensorimotor configuration (sensory input, efference copy, etc.) of a behavior at nearby points in time, adjacent PCs in the model would tend to respond one after another in temporal sequence during a learned behavior. Such a "wave" of PC activity has been observed and simulated by Braitenberg et al. (1997).

Rather than mediating error-driven learning as in the Marr/Albus paradigm, LTD and LTP could mediate unsupervised learning as envisioned for InMin. Before CF spikes, all of the active PF-to-PC synapses in a MZ would increase via LTP, and they would all decrease again at a CF spike via LTD. The CFs simply carry "learn now" signals for InMin. Any mechanism by which the PF weights of selected PCs undergo less LTD than of others in the MZ should produce the specialization required for InMin. PCs are selected through compe- tition in the current version of InMin. Actual competition is not necessary, although it is possible that inhibitory interconnections between PCs, directly via PC axon collaterals and indirectly through inhibitory interneurons, may mediate competitive interactions among PCs (Llinás and Walton 1990; Dunbar et al. 2004). Such a competition could be initiated through the intense activation of PCs by CF spikes. PF-to-PC weight normalization could occur through homeostatic mechanisms intrinsic to single neurons (Turrigiano 1999), or through coordination between PC and inferior olive activities that temporally distributes CF spikes so as to balance LTD and LTP, and keep the net weight of PF input to PCs at a constant level (Kenyon et al. 1998).

Again for simplicity, InMin implements a conventional form of reinforcement learning (Alspector et al. 1993; Venkatesh 1993; Sutton and Barto 1998). Reinforcement learning as envisioned for InMin would be mediated by mechanisms other than LTD and LTP. In the current version of InMin, CF spikes transiently perturb the output weights of all the PCs in a MZ and, if the negative reinforcement signal (i.e., overall PF activity) is reduced, the algorithm makes part of the perturbation permanent. Adjusting the output weights of PCs is equivalent to changing the sensitivity of the cell, and such changes have been reported for PCs (Schreurs et al. 1998). In any case, evidence is building for plasticity of the synaptic connections between PCs and deep cerebellar nuclei neurons (Morishita and Sastry 1996; Aizenman et al. 1998; Ouardouz and Sastry 2000; Ohyama et al. 2006). It is reasonable to assume plasticity of the synapses between PCs and the vestibular nuclei neurons that mediate smooth pursuit.

The model of cerebellar control of pursuit put forth by Kettner et al. (1997) employs an eligibility trace, which compensates for the delay in the retinal slip error signal that their model uses for supervised learning. They suggested that an eligibility trace could be implemented by second messenger systems in PCs (ibid). PCs in the current version of InMin integrate their PF input activities over time, thereby making an eligibility trace unnecessary. However, they still rely on PC second messengers for many functions, including the implementation of two input integrators, one leaking faster than the other, comparison of the states of the integrators, implementation of weight perturbation, and weight adaptation based on the results of the input integrator comparison. While the current version of InMin assumes a substantial amount of second messenger computing, all of these computations are simple (e.g., integration, comparison, addition). The assumption of second messenger computation in InMin is justified because PCs are known for the complexity of their second messenger systems (Linden 1996; Ito 2002), and because the required computations are among those of which second messenger systems are thought to be capable (Katz and Clemens 2001). Recent work provides 
evidence specifically for leaky integrators in PCs (Tanaka et al. 2007).

\subsection{Encoding of pursuit variables by Purkinje cells}

As pointed out by Kettner et al. (1997), the cerebellum contributes a predictive component to pursuit. This is compatible with the idea that the smooth eye movement part of the frontal eye fields (FEFsem) mediates servo control of pursuit, which is retarded by the delay inherent in the visual processing needed to produce the retinal slip error signal. The delay would also limit the gain of the FEFsem contribution, because delay combined with high gain can lead to instability in negative feedback servo controllers (Milsum 1966). The cerebellum, acting as a feedforward pattern correlator (Anastasio 2001b), may both decrease the delay and increase the gain of pursuit.

In the model of Kettner et al. (1997) and in this model, the cerebellum generates its predictive command by combining $\mathrm{PF}$ inputs that have a variety of phase relationships relative to oculomotor variables. In the Kettner model, which follows the Marr/Albus paradigm, the weights of the various PF inputs are determined via supervised learning (see next subsection). In contrast, InMin determines these weights via unsupervised (SOM) learning (see Sect. 2), in which the input patterns are the activities of the PFs at a moment in time that is marked by the occurrence of a CF spike. Each model PC becomes specialized for a distinct pattern of GC (PF) input. As the CF spikes occur at random times, the preferred PF patterns of the PCs span the entire range of possible patterns. The resulting diversity in the temporal and spatial response properties of the model PCs agrees well with observation (Stone and Lisberger 1990a; Leung et al. 2000; Suh et al. 2000; Takemura et al. 2001; Kettner et al. 2002).

The preferred input patterns of model PCs provides the basis of a possible explanation for the puzzling spatiotemporal properties of PCs in the floccular complex. Many PCs in the floccular complex have diagonal spatial preference, and one might expect that their horizontal and vertical components would have the same phase relationship with eye movement. This is not the case. Kettner and coworkers quantified the combinations of eye position and velocity activity that define the phase relationship of each spatial component for populations of PCs (Leung et al. 2000; Suh et al. 2000). They found that these phase relationships varied widely between cells, and that they were generally different for the horizontal and vertical components of the same PC. The InMin model reproduces these findings (see Fig.9). For complex or realworld stimuli, the phases (e.g., position and velocity components) of the GC responses with different preferred directions at any given time are unlikely to be the same (see Fig. 6). PCs that become specialized for a particular pattern of PF input from those GCs will express the direction and phase combi- nations for that pattern, and the population of PCs as a whole will express the variability inherent in the set of patterns.

The PCs develop diverse input pattern preferences, which lead to realistically diverse spatiotemporal properties, because of the unsupervised learning component of InMin. Simulated $\mathrm{PC}$ responses of the same sort would not be expected using supervised learning, such as that specified under the Marr/ Albus paradigm (see Fig. 11). This difference in expected PC response properties forms the basis of the design of an experimental test that should distinguish between InMin and Marr/Albus.

\subsection{Experimentally distinguishing between InMin and Marr/Albus}

In the InMin algorithm, unsupervised learning causes the PCs to develop phase specific responses, and reinforcement learning adjusts the contribution of those responses to pursuit so as to minimize PF activity and thereby reduce error. The result is that the population of PCs develops a range of response phases, even among PCs in the same MZ. The simulation using InMin can be contrasted with one based on Marr/Albus.

Network architecture is the same for the Marr/Albus case as for the InMin case. The difference is in the training algorithm, the role of CFs, and in the PC output weights. For the Marr/Albus case, the PC output weights are fixed. Also, the CFs carry dimension-specific error signals ( $\dot{r}_{\mathrm{h}}$ or $\dot{r}_{\mathrm{V}}$ ), and the PF-to-PC weights are modified using supervised learning, specifically, the delta rule. Since the Purkinje-output weights are fixed, and since each MZ produces only one dimension-specific output, the error gradient at the PCs can be computed by dividing the error at the output by the value of the fixed Purkinje-output weight.

Training using the delta rule occurs by making weight updates that are correlated with the error signal. Since all the $\mathrm{PCs}$ in a $\mathrm{MZ}$ receive the same $\mathrm{CF}$, which carries the retinal slip error signal under the Marr/Albus paradigm, all of the $\mathrm{PC}$ activities in the same MZ following Marr/Albus training have the same phase. Thus, the Marr/Albus paradigm, which is based on supervised learning of PF-to-PC synapses using $\mathrm{CF}$ error signals, predicts that all PCs that receive the same $\mathrm{CF}$ signal should have the same response phase. This prediction is testable using currently available experimental techniques, owing to the neuroanatomy of the innervation of the cerebellum by CFs.

Climbing fibers, which are the axons of neurons with somata located within the inferior olive, each branch out in the cerebellum to innervate seven PCs, on average (Llinás and Walton 1990; Sugihara et al. 2001, 2004; Sugihara 2006). The set of PCs innervated by the same CF constitute a MZ (Andersson and Oscarsson 1978; Oscarsson 1979; Voogd and Bigaré 1980). PCs in the same $\mathrm{MZ}$ receive the same $\mathrm{CF}$ 
signal, by definition. They could be identified experimentally by the precise synchronization of their complex spikes. As the discharges of neurons in the inferior olive can be synchronized, the complex spikes of nearby PCs can also be synchronized even if they are not in the same MZ (Bell and Kawasaki 1972; Llinás and Sasaki 1989; Sasaki et al. 1989; Wylie et al. 1995; Lang et al. 1996, 1999; Lang 2002; Sugihara and Shinoda 2007). It should be possible experimentally to record from two or more PCs with synchronized complex spikes and test their response phases after cerebellar learning. If PCs with synchronized complex spikes do not have the same response phase, then the Marr/Albus paradigm would be called into question and an alternative interpretation, such as InMin, would be justified.

Acknowledgements Supported in part by the Laboratory Directed Research and Development program at Sandia National Laboratories. Sandia is a multiprogram laboratory operated by Sandia Corporation, a Lockheed Martin Company, for the United States Department of Energy's National Nuclear Security Administration under Contract DEAC04-94AL85000.

Open Access This article is distributed under the terms of the Creative Commons Attribution Noncommercial License which permits any noncommercial use, distribution, and reproduction in any medium, provided the original author(s) and source are credited.

\section{References}

Aizenman C, Manis PB, Linden DJ (1998) Polarity of long-term synaptic gain change is related to postsynaptic spike firing as a cerebellar inhibitory synapse. Neuron 21:827-835

Albus JS (1971) A theory of cerebellar function. Math Biosci 10:25-61

Alspector J, Meir R, Yuhas B, Jayakumar A, Lippe D (1993) A parallel gradient descent method for learning in analog VLSI neural networks. In: Hanson SJ, Cowan JD, Giles CL (eds) Advances in neural and information processing systems, vol 5. Morgan Kaufmann, San Francisco pp 836-844

Anastasio TJ (2001a) Input minimization: a model of cerebellar learning without climbing fiber error signals. Neuroreport 12:38253831

Anastasio TJ (2001b) A pattern-correlation model of vestibulo-ocular reflex habituaion. Neural Netw 14:1-22

Anastasio TJ, Gad YP (2007) Sparse cerebellar innervation can morph the dynamics of a model oculomotor neural integrator. J Comput Neurosci 22:239-254

Andersson G, Oscarsson O (1978) Climbing fiber microzones in cerebellar vermis and their projection to different groups of cells in the lateral vestibular nucleus. Exp Brain Res 32:565-579

Barreiro AK, Bronski JC, Anastasio TJ (2008) Bifurcation theory explains waveform variability in a congenital eye movement disorder. J Comput Neurosci 26:321-329

Becker W, Klein HM (1973) Accuracy of saccadic eye movements and maintenance of eccentric eye positions in the dark. Vision Res 13:1021-1034

Bell CC, Kawasaki T (1972) Relations among climbing fiber responses of nearby Purkinje cells. J Neurophysiol 35:155-169

Braitenberg V, Heck D, Sultan F (1997) The detection and generation of sequences as a key to cerebellar function: experiments and theory. Behav Brain Sci 20:229-277
Brodal P (1979) The pontocerebellar projection in the rhesus monkey: an experimental study with retrograde axonal transport of horseradish peroxidase. Neuroscience 4:193-208

Brodal P (1982) Further observations on the cerebellar projections from the pontine nuclei and the nucleus reticularis tegmenti pontis in the rhesus monkey. J Comp Neurol 204:44-55

Butler AB, Hodos W (1996) Comparative vertebrate neuroanatomy: evolution and adaptation. Wiley-Liss, New York 180-200

Chelazzi L, Ghirardi M, Rossi F, Strata P, Tempia F (1990) Spontaneous saccades and gaze holding ability in the pigmented rat. ii. Effects of localized cerebellar lesions. Eur J Neurosci 2:1085-1094

Crandall WF, Keller EL (1985) Visual and oculomotor signals in nucleus reticularis tegmenti pontis in alert monkey. J Neurophysiol 54:1326-1345

Dunbar RL, Chen G, Gao W, Reinert KC, Feddersen R, Ebner TJ (2004) Imaging parallel fiber and climbing fiber responses and their short-term interactions in the mouse cerebellar cortex in vivo. Neuroscience 126:213-227

Eccles JC, Ito M, Szentagothai J (1967) The cerebellum as a neuronal machine. Springer, Berlin

Faulstich M, van Laphen AM, Luo C, du Lac S, De Zeeuw CI (2006) Oculomotor plasticity during vestibular compensation does not depend on cerebellar LTD. J Neurophysiol 96:1187-1195

Fuchs AF (1967) Saccadic and smooth pursuit eye movements in the monkey. J Physiol 191:609-631

Fujita M (1982) Adaptive filter model of the cerebellum. Biol Cybern 45:195-206

Gibson AR, Horn KM, Pong M (2004) Activation of climbing fibers. Cerebellum 3:212-221

Gilman S (1981) Disorders of the cerebellum, Contemporary Neurology Series, vol 21. FA Davis, Philadelphia

Glickstein M, Gerrits N, Kralj-Hans I, Mercier B, Stein J, Voogd J (1994) Visual pontocerebellar projections in the macaque. J Comp Neurol 349:51-72

Goffart L, Chen LL, Sparks DL (2003) Saccade dysmetria during functional perturbation of the caudal fastigial nucleus in the monkey. Ann NY Acad Sci 1004:220-228

Goldberg ME, Segraves MA (1989) The visual and frontal cortices. Rev Oculomot Res 3:283-313

Guitton D, Bergeron A, Choi W-Y, Matsuo S (2003) On the feedback control of orienting gaze shifts made with eye and head movements. Prog Brain Res 142:55-68

Hansel C, Linden DJ, D’Angelo E (2001) Beyond parallel fiber LTD: the diversity of synaptic and non-synaptic plasticity in the cerebellum. Nat Neursci 4:467-475

Hartell NA (2002) Parallel fiber plasticity. Cerebellum 1:3-18

Hirano T (1990) Depression and potentiation of the synaptic transmission between a granule cell and a Purkinje cell in rat cerebellar culture. Neurosci Lett 119:141-144

Ito M (1984) The cerebellum and neural control. Raven Press, New York

Ito M (2000) Mechanisms of motor leraning in the cerebellum. Brain Res 886:237-245

Ito M (2001) Cerebellar long-term depression: characterization, signal transduction, and functional roles. Physiol Rev 81:1143-1195

Ito M (2002) The molecular organization of cerebellar long-term depression. Nat Rev Neurosci 3:896-902

Ito M, Sakurai M, Tongroach P (1982) Climbing fiber induced depression of both mossy fibre responsivemes and glutamate sensitivity of cerebellar Purkinje cells. J Physiol (Lond) 324:113-361

Kahlon M, Lisberger SG (2000) Changes in the responses of Purkinje cells in the floccular complex of monkeys after motor learning in smooth pursuit eye movements. J Neurophysiol 84:2945-2960

Katz PS, Clemens S (2001) Biochemical networks in nervous systems: expanding neuronal information capacity beyond voltage signals. Trends Neurosci 24:18-25 
Kawano K, Shidara M, Watanabe Y, Yamane S (1994) Neural activity in cortical area MST of alert monkey during ocular following responses. J Neurophysiol 71:2305-2324

Keating EG (1991) Frontal eye field lesions impair predictive and visually-guided pursuit eye movements. Exp Brain Res 86:311323

Keating EG (1993) Lesions of the frontal eye field impair pursuit eye movements, but preserve the predictions driving them. Behav Brain Res 53:91-104

Keating JG, Thach WT (1995) Nonclock behavior of inferior olvie neurons: interspike interval of Purkinje cell complex spike discharge in the awake behaving monkey is random. J Neurophysiol 73:1329-1340

Keating JG, Thach WT (1997) No clock signal in the discharge of neurons $\mathrm{n}$ the deep cerebellar nuclei. J Neurophysiol 77:22322234

Kenyon GT, Median JF, Mauk MD (1998) A mathematical model of the cerebellar-olivary system. 1 . Self-regulating equilibrium of climbing fiber activity. J Comput Neurosci 5:17-33

Kettner RE, Leung H-C, Peterson BW (1996) Predictive smooth pursuit of complex two-dimensional trajectories in monkey: component interactions. Exp Brain Res 108:221-235

Kettner RE, Mahamud S, Leung H-C, Sitkoff N, Houk JC, Peterson BW, Barto AG (1997) Prediction of complex two-dimensional trajectories by a cerebellar model of smooth pursuit eye movement. J Neurophysiol 77:2115-2130

Kettner RE, Suh M, Davis D, Leung HC (2002) Modeling cerebellar flocculus and paraflocculus involvement in complex predictive smooth eye pursuit in monkeys. Ann NY Acad Sci 978:455-467

Kitazawa S, Wolpert DM (2005) Rhythmicity, randomness and synchrony in climbing fiber signals. Trends Neurosci 28: 611-619

Kobayashi Y, Kawano K, Takemura A, Inoue Y, Kitama T, Gomi H, Kawato M (1998) Temporal firing patterns of Purkinje cells in the cerebellar ventral paraflocculus during ocular following responses in monkeys. II. Complex spikes. J Neurophysiol 80:832-848

Kohonen T (1997) Self-organizing maps, 2nd edn. Springer-Verlag, Berlin

Krauzlis RJ (2004) Recasting the smooth pursuit eye movement system. J Neurophysiol 91:591-603

Kuroda S, Yamamoto K, Miyamoto H, Doya K, Kawato M (2001) Statistical characteristics of climbing fiber spikes necessary for efficient cerebellar learning. Biol Cybern 84:183-192

Lang EJ (2002) GABAergic and glutamatergic modulation of spontaneous and motor-cortex-evoked complex spike activity. J Neurophysiol 87:1993-2008

Lang EF, Sugihara I, Llinás R (1996) GABAergic modulation of complex spike activity by the cerebellar nucleoolivary pathway in rat. J Neurophysiol 76:255-275

Lang EF, Sugihara I, Welsh JP, Llinás R (1999) Patterns of spontaneous Purkinje cell complex spike activity in the awake rat. J Neurosci 19:2728-2739

Leung H-C, Kettner RE (1997) Predictive smooth pursuit of complex two-dimensional trajectories demonstrated by perturbation response in monkeys. Vision Res 37:1347-1354

Leung HC, Suh M, Kettner RE (2000) Cerebellar flocculus and paraflocculus Purkinje cell activity during circular pursuit in monkey. J Neurophysiol 83:13-30

Lev-Ram V, Mehta SB, Kleinfeld D, Tsien RY (2003) Reversing cerebellar long-term depression. Proc Natl Acad Sci 100(26): 1598915993

Linden DJ (1996) Cerebellar long-term depression as investigated in a cell culture preparation. Behav Brain Sci 19:339-346

Llinás R, Sasaki K (1989) The functional organization of the olivocerebellar system as examined by multiple Purkinje cell recordings. Eur J Neurosci 1:587-602
Llinás RR, Walton KD (1990) Cerebellum. In: Shepherd GM (ed) The synaptic organization of the brain. Oxford University Press, New York pp 214-245

Lynch JC (1987) Frontal eye field lesions in monkeys disrupt visual pursuit. Exp Brain Res 68:437-441

MacAvoy MG, Gottlieb JP, Bruce CJ (1991) Smooth-pursuit eye movement representation in the primate frontal eye field. Cereb Cortex 1:95-102

Marr D (1969) A theory of cerebellar cortex. J Physiol (Lond) 202: 437-470

Miles FA, Fuller JH (1975) Visual tracking the primate flocculus. Science 189:1000-1002

Milsum JH (1966) Biological control systems analysis. McGraw-Hill, New York

Morishita W, Sastry BR (1996) Postsynaptic mechanisms underlying long-term depression of GABAertic transmission in neurons of the deep cerebellar nuclei. J Neurophysiol 76:59-68

Mustari MJ, Fuchs AF, Wallman J (1988) Response properties of dorsolateral pontine units during smooth pursuit $\mathrm{n}$ the rhesus monkey. J Neurophysiol 60:664-686

Newsome WT, Wurtz RH, Komatsu H (1988) Relation of cortical areas MT and MST to pursuit eye movements. II. differentiation of retinal from extraretinal inputs. J Neurophysiol 60:604-620

Nolte J (2002) The human brain: an introduction to its functional anatomy, 5th edn. Mosby/Harcourt, St. Louis

Ohyama T, Nores WL, Medina JF, Riusech FA, Mauk MD (2006) Learning-induced plasticity in deep cerebellar nucleus. J Neurosci 26:12656-12663

Oscarsson O (1979) Functional units of the cerebellum: saggital zones and microzones. Trends Neurosci 2:143-145

Ouardouz M, Sastry BR (2000) Mechanisms underlying LTP of inhibitory synaptic transmission in the deep cerebellar nuclei. J Neurophysiol 84:1414-1421

Rambold H, Churchland A, Selig Y, Jasmin L, Lisberger SG (2002) Partial ablation of the flocculus and ventral paraflocculus in monkeys cause linked deficits in smooth pursuit eye movements and adaptive modification of the VOR. J Neurophysiol 87: 912-924

Robinson DA (1974) The effect of cerebellectomy on the cat's vestibule-ocular integrator. Brain Res 71:195-207

Robinson DA (1981) The use of control systems analysis in the neurophysiology of eye movements. Ann Rev Neurosci 4:463-503

Ron S, Robinson DA (1973) Eye movements evoked by cerebellar stimulation in the alert monkey. J Neurophysiol 36:1004-1022

Sakurai M (1987) Synaptic modification of parallel fibre-Purkinje cell transmission in in-vitro guinea-pig cerebellar slices. J Physiol (Lond) 394:463-480

Sasaki K, Bower JM, Llinás R (1989) Multiple Purkinje cell recording in rodent cerebellar cortex. Eur J Neurosci 6:572-586

Schreurs BG, Gusev PA, Tomsic D, Aldon DL, Shi T (1998) Intracellular correlates of acquisition and long-term memory of classical conditioning in Purkinje cell dendrites in slices of rabbit cerebellar lobule HVI. J Neurosci 18:5498-5507

Shi D, Friedman HR, Bruce CJ (1998) Deficits in smooth-pursuit eye movements after muscimol inactivation within the primate's frontal eye fields. J Neurophysiol 80:458-464

Simpson JI, Wylie DR, De Zeeuw CI (1996) On climbing fiber signals and their consequence(s). Behav Brain Sci 19:384-410

Simpson JI, Belton T, Suh M, Winkelman B (2002) Complex spike activity in the flocculus signals more than the eye can see. Ann NY Acad Sci 978:232-236

Sparks DL, Barton EJ (1993) Neural control of saccadic eye movements. Curr Opin Neurobiol 3:966-972

Stone LS, Lisberger SG (1990a) Visual responses of Purkinje cells in the cerebellar flocculus during smooth-pursuit eye movements in monkeys. i. simple spikes. J Neurophysiol 63:1241-1261 
Stone LS, Lisberger SG (1990b) Visual responses of Purkinje cells in the cerebellar flocculus during smooth-pursuit eye movements in monkeys. ii. complex spikes. J Neurophysiol 63:1262-1275

Sugihara I (2006) Organization and remodeling of the olivocerebellar climbing figher projection. Cerebellum 5:15-22

Sugihara I, Shinoda Y (2007) Molecular, topographical, and functional organization of the cerebellar nuclei: analysis by threedimensional mapping of the olivonuclear projection and aldolase C labeling. J Neurosci 27:9696-9710

Sugihara I, Wu HS, Shinoda Y (2001) The entire trajectories of single olivocerebellar axons in the cerebral cortex and their contribution to cerebellar compartmentalization. J Neurosci 21:7715-7723

Sugihara I, Ebata S, Shinoda Y (2004) Funcitonal compartmentalization in the flocculus and the ventral dentate and dorsal group y nuclei: an analysis of single olivocerebellar axonal morphology. J Comp Neurol 470:113-133

Suh M, Leung H-C, Kettner RE (2000) Cerebellar flocculus and ventral paraflocculus Purkinje cell activity during predictive and visually driven pursuit in monkey. J Neurophysiol 84:1835-1850

Sutton R, Barto A (1998) Reinforcement learning: an introduction. MIT Press, Cambridge, MA

Suzuki DA, May JG, Keller EL, Yee RD (1990) Visual motion response properties of neurons in dorsolateral pontine nucleus of alert monkey. J Neurophysiol 63:37-59

Tain JR, Lynch JC (1996a) Functionally defined smooth and saccadic eye movement subregions in the frontal eye field of Cebus monkeys. J Neuorphysiol 76:2740-2753

Tain JR, Lynch JC (1996b) Corticocortical input to the smooth and saccadic eye movement subregions of the frontal eye field in Cebus monkeys. J Neurophysiol 76:2754-2771

Takagi M, Zee DS, Tamargo RJ (1998) Effects of lesions of the oculomotor vermis on eye movements in primate: saccades. J Neurophysiol 80:1911-1931

Takemura A, Inoue Y, Gomi H, Kawato M, Kawano K (2001) Change in neuronal firing patterns in the process of motor command generation for the ocular following response. J Neurophysiol 86: 1750-1763

Tanaka M, Fukushima K (1998) Neuronal responses related to smooth pursuit eye movements in the periarcuate cortical area of monkeys. J Neurophysiol 80:28-47
Tanaka M, Lisberger SG (2001) Regulation of the gain of visuallyguided smooth pursuit eye movements by frontal cortex. Nature 409:191-194

Tanaka M, Lisberger SG (2002) Enhancement of multiple components of pursuit eye movement by microstimulation in the arcuate frontal pursuit area in monkeys. J Neurophysiol 87:802-818

Tanaka K, Khiroug L, Santamaria F, Doi T, Ogasawara H, Ellis-Davies GC, Kawato M, Augustine GJ (2007) Ca2+ requirements for cerebellar long-term synaptic depression: role for a postsynaptic leaky integrator. Neuron 54:787-800

Turrigiano GC (1999) Homeostatic plasticity in neuronal networks: the more things change, the more they stay the same. Trends Neurosci 22:221-227

van Alphen AM, De Zeeuw CI (2002) Cerebellar LTD facilitates but is not essential for long-term adaptation of the vestibule-ocular reflex. Eur J Neurosci 16:486-490

Venkatesh SS (1993) Directed drift: a new linear threshold algorithm for learning binary weights on-line. J Comp Syst Sci 46:198-217

Voogd J, Bigare F (1980) Topographical distribution of olivary and cortico-nulcear fibers in the cerebellum: a review. In: Courville J, de Montigny C, Lamarre Y (eds) The inferior olivary nucleus: anatomy and physiology. Raven, New York

Welsh JP, Yamaguchi J, Zeng X-H, Kojo M, Nakada Y, Takagi A, Sugimori M, Llinas RR (2005) Normal motor learning during pharmacological prevention of Purkinje cell long-term depression. Proc Natl Acad Sci 102:17166-17171

Widrow B, Hoff ME (1960) Adaptive switching circuits. In: 1960 IRE WESCON Convention Record, Part 4, New York, pp 96-104

Wylie DR, De Zeeuw CI, Simpson JI (1995) Temporal relations of the complex spike activity of Purkinje cell pairs in the vestibulocerebellum of rabbits. J Neurosci 15:2875-2887

Yamamoto K, Kobayashi Y, Takemura A, Kawano K, Kawato M (2002) Computational studies on acquisition and adaptation of ocular following responses based on cerebellar synaptic plasticity. J Neurophys 87:1554-1571

Zee DS, Yamazaki A, Butler PH, Gucer G (1981) Effects of ablation of flocclus and paraflocculus on eye movements in primate. J Neurophysiol 46:878-899 